\title{
Manipulation of a VEGF-Notch signaling circuit drives formation of functional vascular endothelial progenitors from human pluripotent stem cells
}

\author{
Makoto Sahara $^{1,2,3,4}$, Emil M Hansson ${ }^{1,2}$, Oliver Wernet ${ }^{5}$, Kathy O Lui ${ }^{1,2,3}$, Daniela Später ${ }^{1,2,3}$, Kenneth R Chien ${ }^{1,2,4}$ \\ ${ }^{I}$ Department of Stem Cell and Regenerative Biology, ${ }^{2}$ Harvard Stem Cell Institute, Harvard University, 7 Divinity Avenue, Cam- \\ bridge, MA 02138, USA; ${ }^{3}$ Center for Regenerative Medicine, Massachusetts General Hospital, 185 Cambridge Street, Boston, \\ MA 02114, USA; ${ }^{4}$ Department of Medicine-Cardiology/Cell and Molecular Biology, Karolinska Institutet, SE-171 77 Stockholm, \\ Sweden; ${ }^{5}$ Department of Anesthesiology and Intensive Care Medicine, Charité-University Medicine Berlin, Campus Charité Mitte, \\ Charitéplatz 1, 10117 Berlin, Germany
}

Human pluripotent stem cell (hPSC)-derived endothelial lineage cells constitutes a promising source for therapeutic revascularization, but progress in this arena has been hampered by a lack of clinically-scalable differentiation protocols and inefficient formation of a functional vessel network integrating with the host circulation upon transplantation. Using a human embryonic stem cell reporter cell line, where green fluorescent protein expression is driven by an endothelial cell-specific VE-cadherin (VEC) promoter, we screened for $>60$ bioactive small molecules that would promote endothelial differentiation, and found that administration of BMP4 and a GSK-3 $\beta$ inhibitor in an early phase and treatment with VEGF-A and inhibition of the Notch signaling pathway in a later phase led to efficient differentiation of hPSCs to the endothelial lineage within six days. This sequential approach generated $>50 \%$ conversion of hPSCs to endothelial cells (ECs), specifically $\mathrm{VEC}^{+} \mathrm{CD} 31^{+} \mathrm{CD} 34^{+} \mathrm{CD} 14^{-} \mathrm{KDR}^{\text {high }}$ endothelial progenitors (EPs) that exhibited higher angiogenic and clonogenic proliferation potential among endothelial lineage cells. Pharmaceutical inhibition or genetical knockdown of Notch signaling, in combination with VEGF-A treatment, resulted in efficient formation of EPs via $\mathrm{KDR}^{+}$mesodermal precursors and blockade of the conversion of EPs to mature ECs. The generated EPs successfully formed functional capillary vessels in vivo with anastomosis to the host vessels when transplanted into immunocompromised mice. Manipulation of this VEGF-A-Notch signaling circuit in our protocol leads to rapid large-scale production of the hPSC-derived EPs by 12- to 20-fold vs current methods, which may serve as an attractive cell population for regenerative vascularization with superior vessel forming capability compared to mature ECs.

Keywords: embryonic stem cell; endothelial progenitor; Notch signaling; VEGF

Cell Research (2014) 24:820-841. doi:10.1038/cr.2014.59; published online 9 May 2014

\section{Introduction}

Therapeutic vascularization through transplantation of endothelial lineage cells constitutes a conceptually

Correspondence: Makoto Sahara ${ }^{\mathrm{a}}$, Kenneth R Chien ${ }^{\mathrm{b}}$

${ }^{a}$ Tel: +468524 874 79; Fax: +468311101

E-mail: makoto.sahara@ki.se

${ }^{\text {b}}$ Tel: +46 8524874 67; Fax: +46 8311101

E-mail: kenneth.chien@ki.se

Received 23 December 2013; revised 10 March 2014; accepted 31 March 2014; published online 9 May 2014 attractive treatment for solid organs with pathology affecting the local circulation, such as the ischemic heart $[1,2]$. Human embryonic stem cells (hESCs) and human inducible pluripotent stem cells (hiPSCs), collectively referred to as human pluripotent stem cells (hPSCs), can be expanded indefinitely and have the potential to differentiate to virtually any cell type in the human body $[3,4]$, thereby offering a plentiful source of cells for cell-based therapy approaches, including vascularization using endothelial cells (ECs) $[2,5]$. However, a critical and frequently overlooked problem is the challenge of obtaining a sufficient number of cells for transplantation 
$[2,5-9]$. Differentiation protocols permitting isolation of a cell type of interest with high purity and yield are often not available, and the desired cell type is generally a terminally differentiated cell with low capacity for proliferation. There is growing appreciation in the field that isolation and expansion of committed progenitor cells, characterized by a restriction in developmental potential and a high proliferative rate, offers a potential remedy to these challenges. Previous reports, including from our laboratory, have documented the validity of this approach for the heart [10] and endocrine pancreatic [11] cell lineages. In the present study, we have extended this concept to cells of the endothelial lineage. We present a differentiation protocol resulting in rapid and efficient differentiation of hPSCs to the endothelial lineage, and have isolated and purified endothelial progenitors (EPs), a committed progenitor population within this lineage [12-14]. Moreover, we have identified the key molecular mechanisms controlling the balance between maintenance of progenitor identity and further differentiation and maturation along the $\mathrm{EC}$ lineage.

We performed a screen in an effort to optimize differentiation of hPSCs into endothelial lineage cells, with a focus specifically on identifying an EP population. By screening $>60$ bioactive molecules, we identified two distinct phases during differentiation of hPSCs to the endothelial lineage. Priming cultures in the early phase with bone morphogenic protein (BMP) 4 and a glycogen synthase kinase- $3 \beta$ inhibitor (GSK-3 $\beta \mathrm{I}$ ), and EP induction in a later phase with vascular endothelial growth factor (VEGF)-A and pharmacological suppression of the Notch signaling pathway by the $\gamma$-secretase inhibitor DAPT, synergistically enhanced hPSC differentiation into EPs, defined as VE-cadherin (VEC) ${ }^{+} \mathrm{CD} 31^{+} \mathrm{CD} 34^{+} \mathrm{CD} 14^{-}$ cells. This cellular population exhibited the highest angiogenic and proliferative potential in clonal assays among the generated endothelial lineage cells, and further transcriptional analyses identified them as a population exhibiting an EP-specific molecular profile such as $\mathrm{KDR}^{\text {high }} \mathrm{Id}{ }^{\text {high }}$ Neuropilin ${ }^{\text {high }}$, which was similar to that of human umbilical cord blood cells (HUCBCs) [12-14]. Mechanistic analyses revealed that BMP4 with GSK-3 $\beta$ I enhanced KDR promoter activity and thereby the induction of $\mathrm{KDR}^{+}$mesodermal precursors. VEGF-A was identified as an essential factor for endothelial differentiation from such $\mathrm{KDR}^{+}$mesodermal precursors; however, VEGF-A alone also promoted EP $\left(\mathrm{VEC}^{+} \mathrm{KDR}^{\text {high }} \mathrm{CD} 34^{+}\right)$ differentiation into mature $\mathrm{ECs}\left(\mathrm{VEC}^{+} \mathrm{KDR}^{\text {low }} \mathrm{CD} 34^{-}\right)$, or $\mathrm{KDR}^{+}$precursor differentiation into non-endothelial lineage cells, e.g., smooth muscle cells (SMCs), which was enhanced especially in cultures with active delta-like 4 (D114)/Notch signaling. In contrast, inhibition of Notch signaling by DAPT or genetic knockdown of core components of the pathway using shRNA maintained high levels of KDR promoter activity, thereby promoting EP proliferation and blocking further differentiation to mature ECs. Therefore, our study showed that the combined use of DAPT with VEGF-A in a late phase promotes the efficient generation and proliferation of $\mathrm{KDR}^{+}$precursorderived EPs.

Using this sequential approach (priming with BMP4 and GSK-3 3 I, induction with VEGF-A and DAPT), hPSCderived EPs could be generated in only 6 days with around $50 \%$ efficiency. hPSC-derived EPs successfully formed functional capillary vessel networks with anastomoses to host vessels in vivo after engraftment into immunocompromised mice, and also improved heart function in mice after myocardial infarction (MI). To our knowledge, this is the first description of a quick and efficient method for large-scale production of hPSC-derived EPs, and such cells are a promising cellular source for therapeutic revascularization in ischemic cardiovascular diseases and in drug screening for compounds facilitating therapeutic angiogenesis and vasculogenesis.

\section{Results}

Transgenic hESC reporter cell line for monitoring of endothelial differentiation

We established a transgenic reporter hESC line for convenient monitoring of differentiation to the endothelial lineage. A 2.5-kilobase promoter sequence of the ECspecific VEC (CDH5) gene $[15,16]$ was inserted into a lentiviral vector upstream of a cDNA sequence encoding enhanced green fluorescent protein (EGFP) (VEC-EGFP; Figure 1A). Lentiviral particles were produced and used to transduce human primary ECs, human primary foreskin fibroblasts, and human primary SMCs. Transduced ECs exhibited robust expression of EGFP, whereas no EGFP expression could be detected in transduced fibroblasts or SMCs (Supplementary information, Figure S1A-S1D). WA09 hESCs were transduced with VECEGFP lentiviral particles and individual clones were selected and expanded. After spontaneous differentiation, six clonally expanded lines exhibited co-expression of EGFP and the pan-EC marker CD31 (or endogenous VEC) (Figure 1B, 1C and Supplementary information, Figure S1E), and one hESC-VEC-EGFP reporter line was selected for subsequent experiments. hESCderived EGFP-expressing cells did not express alphasmooth muscle actin or vimentin, a marker of fibroblasts (Supplementary information, Figure S1F and S1G). The differentiated VEC-EGFP ${ }^{+}$cells sorted by fluorescenceactivated cell sorting (FACS) proliferated rapidly after 
A
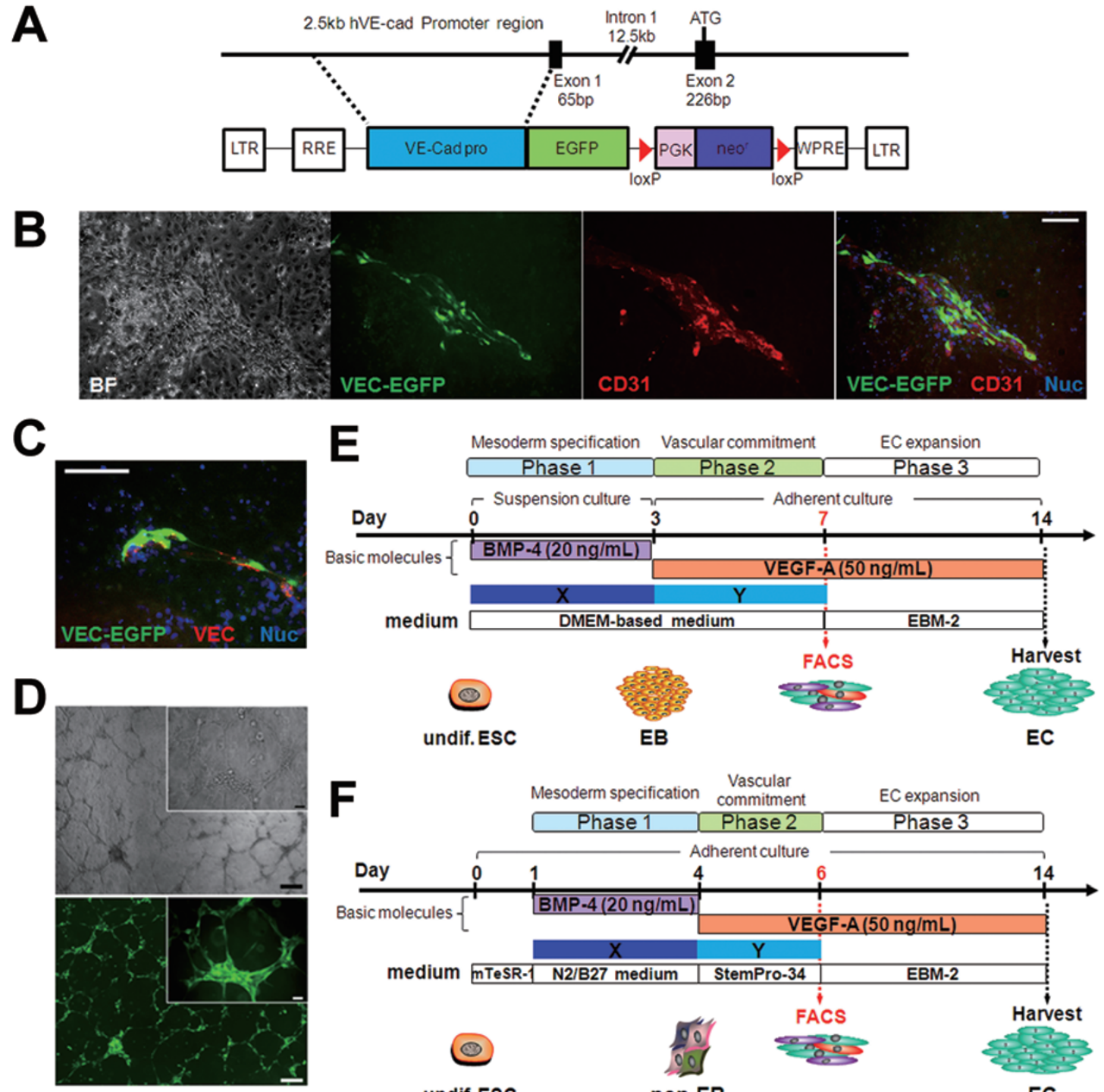

G
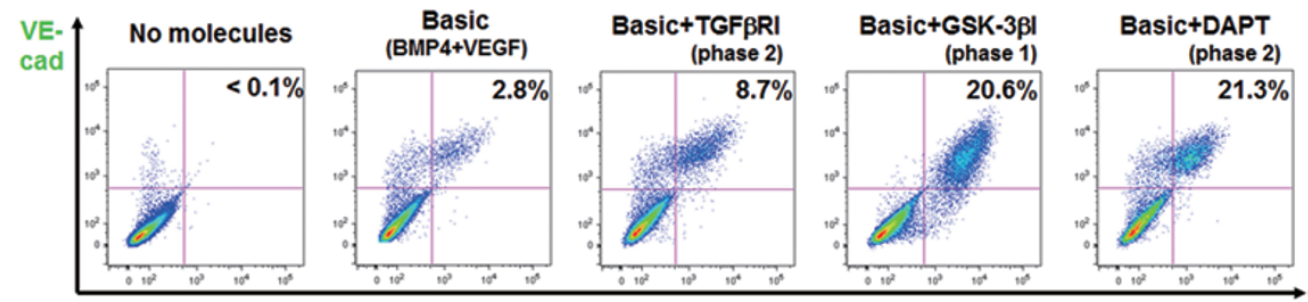

H
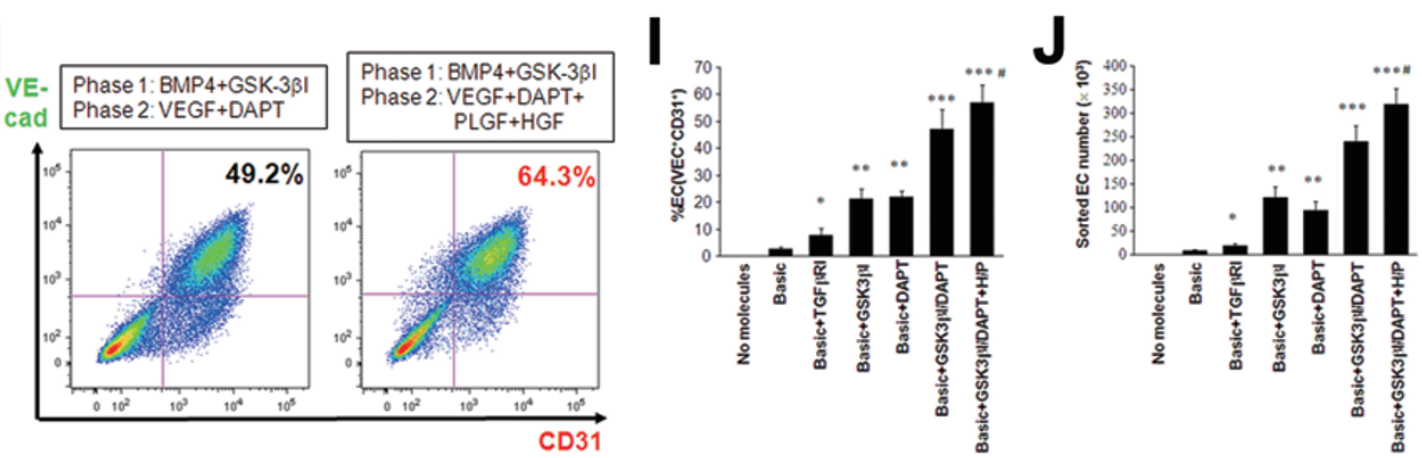
replating (Supplementary information, Figure $\mathrm{S} 1 \mathrm{H}$ ) and formed capillary-like structures in vitro (Figure 1D). Immunocytochemistry revealed that VEC-EGFP ${ }^{+}$cells expressed the well-characterized EC markers CD31, VEC and von Willbrand factor (vWF) (Supplementary information, Figure S1I). Taken together, these data document that the VEC promoter construct faithfully reports VEC expression, and that transgenic VEC-EGFP hESC lines express EGFP as they adopt the EC fate.

\section{Optimization of differentiation to the endothelial lineage}

We used the transgenic VEC-EGFP reporter hESC line to optimize endothelial differentiation of hESCs. Differentiation experiments were performed using two previously described protocols with minor modifications: one based on embryoid body (EB) formation in suspension culture (method A) [17], and the other based on monolayers of dissociated adherent single cells (method B) [18] (Figure 1E and 1F). Both protocols are comprised of three steps: mesodermal specification (phase 1), vascular commitment (phase 2), and EC expansion after sorting the VEC-EGFP ${ }^{+}$endothelial population using FACS (phase 3). We found treatment with BMP4 in phase 1 and VEGF-A in phase 2 to be indispensable for endothelial differentiation. No $\mathrm{VEC}^{+} \mathrm{CD} 31^{+} \mathrm{ECs}$ were detected when either of these factors was omitted. However, as previously reported [19-21], the efficiency of endothelial induction with BMP4/VEGF-A was only $1 \%-3 \%$ of the total cell population on day 6-7, resulting in a low yield of ECs (Supplementary information, Figure S2A and S2B). Using a library of $>60$ bioactive molecules, consisting of growth factors, cytokines, and activators or in- hibitors of signaling pathways (Supplementary information, Table S1), we performed a screen to identify molecules that would enhance BMP4/VEGF-A-mediated endothelial differentiation when administered during phase 1 or 2 of differentiation (Figure $1 \mathrm{E}$ and $1 \mathrm{~F}$ ). Treatment with the GSK-3 $\beta$ inhibitors BIO (6-bromoindirubin-3'oxime; Sigma) or CP21R7 (Roche) in phase 1, resulting in activation of the canonical Wnt signaling pathway, and the $\gamma$-secretase inhibitor DAPT (Sigma) in phase 2, which inhibits the Notch signaling pathway, significantly promoted hESC differentiation into $\mathrm{VEC}^{+} \mathrm{CD} 31^{+} \mathrm{ECs}$ on day 6-7 (Figure 1G and Supplementary information, Figure $\mathrm{S} 2 \mathrm{C}$ and S2D). Application of the TGF $\beta$ signaling inhibitor SB431542 in phase 2 also enhanced hESC endothelial differentiation, as previously reported [17]; however, the efficiency was much lower compared with GSK-3ßI or DAPT treatment (Figure 1G). BMP4/VEGF-A treatment combined with administration of GSK-3I in phase 1 and DAPT in phase 2 was found to enhance endothelial differentiation in a synergistic manner. $\mathrm{VEC}^{+} \mathrm{CD} 31^{+} \mathrm{ECs}$ constituted around $50 \%$ of the total cells and the yield of $\mathrm{VEC}^{+} \mathrm{CD} 31^{+} \mathrm{ECs}$ at the end of phase 2 was increased by 30 -fold (Figure $1 \mathrm{H}-1 \mathrm{~J}$ ). In addition, treatment with placental growth factor (PLGF) and hepatocyte growth factor (HGF) in phase 2 further promoted endothelial differentiation (Figure 1H-1J and Supplementary information, Figure S2E). We did not observe synergistic effects for endothelial differentiation through simultaneous treatment with SB431542 and either GSK-3I or DAPT. As shown in Supplementary information, Figure S2C and S2D, we found that monolayer-based differentiation resulted in more efficient endothelial differentiation and

Figure 1 Establishment of a hESC reporter line for endothelial cell-specific lineage detection and the two modified protocols for endothelial differentiation. (A) A human VE-cadherin (VEC)-targeting construct. A 2.5-kb fragment of the human VE-cadherin promoter region $[15,16]$ was placed upstream of a cDNA encoding EGFP in a lentiviral expression vector (VEC-EGFP). (B, C) Spontaneously differentiating VEC-EGFP hESCs co-expressed EGFP (green) and the pan-endothelial cell marker CD31 (B; red), or endogenous VEC (C; red). BF, brightfield; Nuc, nuclei. Scale bars, $100 \mu \mathrm{m}$. (D) The VEC-EGFP ${ }^{+}$cells could successfully form capillary-like tube structures on Matrigel. Top, brightfield; bottom, VEC-EGFP. Scale bars, $50 \mu \mathrm{m}$ (insets) and $200 \mu \mathrm{m}$. (E, F) Schematic diagrams of the two differentiation protocols. In method A (E), hESC-derived EBs were initially generated in suspension cultures with BMP4 (day 0-3; phase 1), and the EBs were transferred to adherent conditions on day 3 and cultured with VEGF-A (day 3-7; phase 2). In method B (F), dissociated single hESCs were directly seeded on adherent conditions and stimulated with BMP4 (day 1-4; phase 1) in N2/B27 medium, and the medium was switched to StemPro-34 medium containing VEGF-A on day 4 (day 4-6; phase 2). FACS analyses were performed to sort a VEC-EGFP ${ }^{+}$CD31 ${ }^{+}$endothelial cell population on day 7 (method A; E) or day 6 (method B; F), and the sorted cells were expanded in endothelial cell conditions in phase 3 ( day 14). We tested the efficacies of $>60$ bioactive molecules for effects on endothelial differentiation when they were administered in phase 1 (illustrated as " $X$ ") or phase 2 (illustrated as " $Y$ ") in addition to the basic protocol consisting of BMP4 in phase 1 and VEGF-A in phase 2. (G) Representative FACS results detecting the VEC-EGFP CD31 ${ }^{+}$ ECs at the end of phase 2 when the effective molecules for endothelial differentiation were used. $(\mathbf{H})$ The combination of BMP4/GSK-3 $\beta I$ in phase 1 and VEGF-A/DAPT in phase 2 (left) with adjunctive PLGF and HGF in phase 2 (right) synergistically promoted endothelial differentiation and could generate VEC ${ }^{+} \mathrm{CD} 31^{+}$ECs with $\sim 50 \%-60 \%$ efficacy. (I, J) Averaged percent EC (I) and sorted EC number $(\mathrm{J})$ at the end of phase 2 in each treatment combination. $* P<0.01$ and $* * P<0.0001$ vs

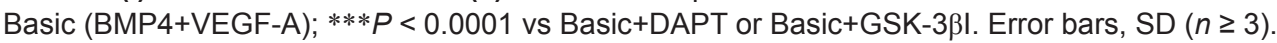


a higher yield of ECs than EB-based differentiation, and therefore all subsequent experiments were performed using the monolayer differentiation protocol. We also found that CP21R7 had more stable results unaffected by its lot number, compared to BIO, and therefore, we basically used CP21R7 as a GSK-3 $\beta$ inhibitor in phase 1 in the following experiments. Experiments in additional hPSC lines verified that our monolayer differentiation protocol, consisting of BMP4/GSK-3 $\beta$ I administration in phase 1 and VEGF-A/DAPT administration in phase 2, resulted in robust endothelial differentiation with minimal differences in efficiency between different cell lines that did not reach statistical significance (Supplementary information, Figure S2F).

BMP4 and a GSK-3 $\beta$ inhibitor enhances the induction of $K D R^{+}$precursors prior to vascular commitment

During endothelial differentiation of hESCs, expression levels of KDR (also known as VEGFR2), a receptor of VEGF-A and a key component of the signaling pathway that specifies EC precursors at their earliest stages of development [22-25], were monitored by flow cytometry. On day 0 , cultures of undifferentiated hESCs exhibited partial and weak expression of KDR and robust expression of TRA-1-60, a marker of undifferentiated hESC (Supplementary information, Figure S3A). During differentiation, $\mathrm{KDR}^{+}$expression levels initially decreased, but increased again on day 4 with stronger intensity after BMP4 treatment (Supplementary information, Figure S3A and S3B), as previously reported $[26,27]$. Intriguingly, the administration of GSK-3 $\beta I$ with BMP4 in phase 1 significantly increased the $\mathrm{KDR}^{+}$ cell ratio on day 4 (Supplementary information, Figure $\mathrm{S} 3 \mathrm{C})$. Either $\mathrm{VEC}^{+}$or $\mathrm{CD} 31^{+}$cells were not detected on day 4; however, they started to emerge with VEGF-A treatment on day 5 (Supplementary information, Figure S3B). To determine the relevance of KDR induction for endothelial differentiation, $\mathrm{KDR}^{+}$and $\mathrm{KDR}^{-}$cells were sorted on day 4 and cultured separately with VEGF-A for 4 days. Flow cytometry analysis revealed that $\sim 60 \%$ of the $\mathrm{KDR}^{+}$cell population differentiated into $\mathrm{VEC}^{+} \mathrm{CD} 31^{+}$ ECs, whereas only less than $1 \%$ of the $\mathrm{KDR}^{-}$cell population became ECs (Supplementary information, Figure S3D). In addition, there was a strong positive correlation between $\mathrm{KDR}^{+}$cell ratio on day 4 and $\mathrm{VEC}^{+}$cell ratio on day 6 (Supplementary information, Figure S3E). These data suggest that $\mathrm{KDR}^{+}$precursors are indispensable for endothelial differentiation of hPSCs and that GSK-3 $\beta$ I with BMP4 enhanced induction of $\mathrm{KDR}^{+}$precursors in phase 1 prior to vascular commitment, likely leading to more efficient endothelial differentiation by the following treatment with VEGF-A in phase 2.
$\mathrm{KDR}^{\text {high }} \mathrm{CD} 34^{+} \mathrm{CD} 14^{-}$subpopulation of $\mathrm{VEC}^{+} \mathrm{CD} 31^{+} \mathrm{ECS}$ exhibits characteristics of EPS

hESC-derived $\mathrm{VEC}^{+}$cells sorted on day 6 co-expressed CD31 and KDR, and the majority $(60 \%-75 \%)$ were also expressing CD34 (Figure 2A). The CD34 antigen, a marker of human hematopoietic stem cells, has been reported to likely label an EP population in adult circulation [12-14, 28-31]. Furthermore, previous work has suggested further subdivision of $\mathrm{CD} 34^{+}$EPs on the basis of expression of the monocytic marker CD14 [24, $32,33]$. We hypothesized that there should be a similar $\mathrm{EP}$ population in the hPSC-derived endothelial lineage, and thereby classified $\mathrm{VEC}^{+} \mathrm{CD} 31^{+}$ECs on day 6 into three subpopulations by the presence or absence of CD34 and CD14 for further analyses examining EP characteristics of these subpopulations (Figure 2B). Before that, we confirmed mRNA expression of CD14 in only CD14 ECs sorted by FACS with RT-PCR, but not in CD14 ECs (Figure 2C).

Among the three EC subpopulations, $\mathrm{CD} 34^{+} \mathrm{CD} 14$ ECs showed the highest vascular tube formation potency in vitro, comparable to that of HUCBCs, which are known to include immature EPs to a higher degree (Figure 2D-2F) [12-14]. CD34 ${ }^{+} \mathrm{CD} 14^{-}$ECs also exhibited the highest proliferative activity (Figure 2G). In keeping with this, $\mathrm{CD} 34^{+} \mathrm{CD} 14^{-} \mathrm{ECs}$ generated the largest number of $\mathrm{VEC}^{+} \mathrm{CD} 31^{+} \mathrm{ECs}$ at the end of phase 3 (day 14), when each subpopulation was separately cultured (Figure $2 \mathrm{H}$ ). To analyze molecular profiles of these EC subpopulations compared to HUCBCs and human umbilical vein ECs (HUVECs), PCR array analyses were performed focusing on endothelial lineage-related genes (Figure 2I and $2 \mathrm{~J})$. As shown in Figure 2I, endothelial lineage-enriched genes such as CD31 (PECAM1), endoglin (ENG), VEC $(C D H 5)$, and endothelial nitric oxide synthase (eNOS; also known as NOS3) were approximately equally expressed in all of these groups. Genes that exhibited higher expression levels in $\mathrm{CD} 34^{+} \mathrm{CD} 14^{-}$ECs than in CD34 CD14 ${ }^{-}$and $\mathrm{CD} 14^{+}$ECs included KDR, Flt1 (VEGFR1), $I d 1$, and Neuropilin (NRP), which have been reported as cell surface markers or transcriptional factors expressed in EPs [32-37]. As a result, $\mathrm{CD} 34^{+} \mathrm{CD} 14^{-}$ECs exhibited an expression profile similar to that of HUCBCs, while $\mathrm{CD} 34^{-} \mathrm{CD} 14^{-} \mathrm{ECs}$ had a profile relatively close to that of HUVECs. As expected, CD14 ${ }^{+}$ECs showed inflammatory phenotype, at least in part. We also analyzed molecular profiles of $\mathrm{CD} 34^{+} \mathrm{CD} 14^{-}$EC-derived cells through several passages 30 and 60 days after initial FACS sorting. Interestingly, $\mathrm{CD} 34^{+} \mathrm{CD} 14^{-} \mathrm{EC}$-derived cells after several passages could hold the endothelial lineage phenotype, represented by expression of PECAM1, ENG, CDH5, and NOS3 $[24,32,33]$, although expression of 
EP-related genes had already been decreased (Figure 2I and $2 \mathrm{~J}$ ).

To further test the clonogenic proliferative potential of the three EC subpopulations in a rigorous manner, we developed a clonal single-cell culture assay (Figure $3 \mathrm{~A})$. The percentage of single cells undergoing at least one cell division during 14 days was significantly higher in $\mathrm{CD} 34^{+} \mathrm{CD} 14^{-} \mathrm{ECs}(48.2 \% \pm 6.3 \%)$ than in $\mathrm{CD} 34$
A<smiles>[AlH2]</smiles>

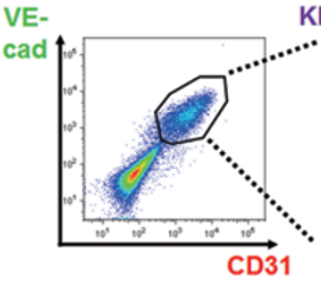

C CD14 GAPDH

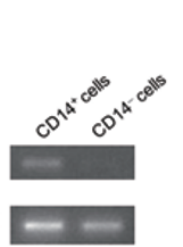

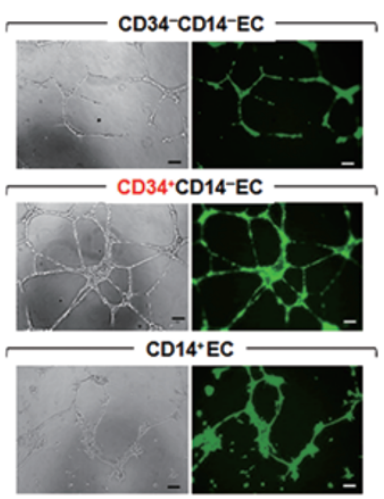
KDR

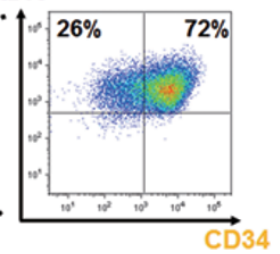

B
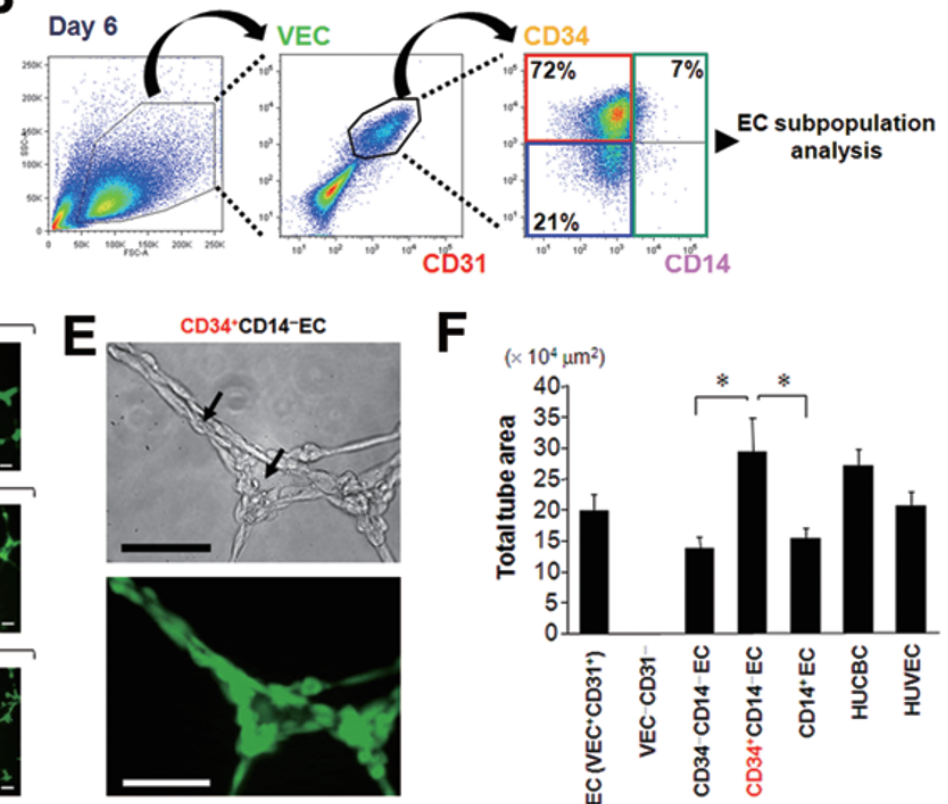

$\mathbf{F}$

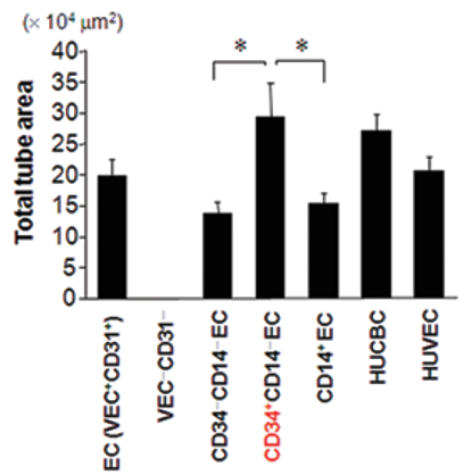

G

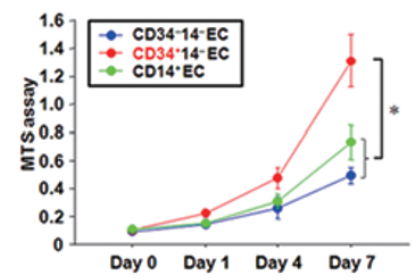

H

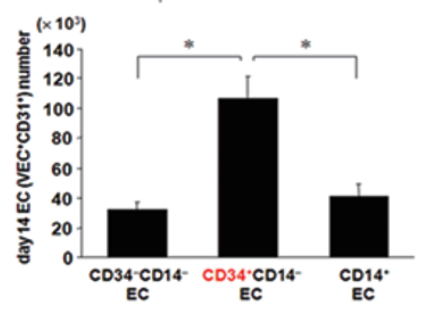

I

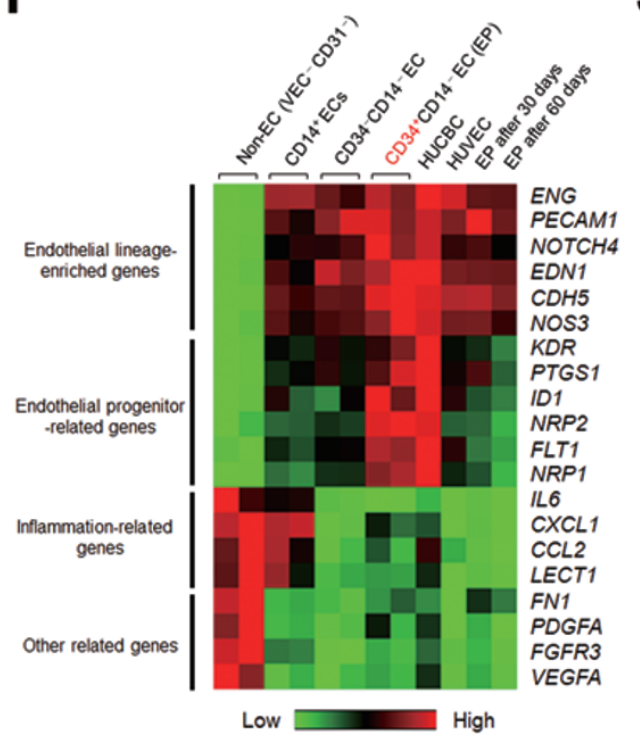

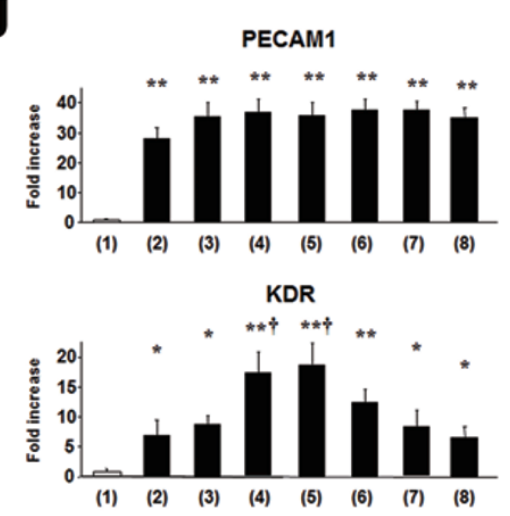
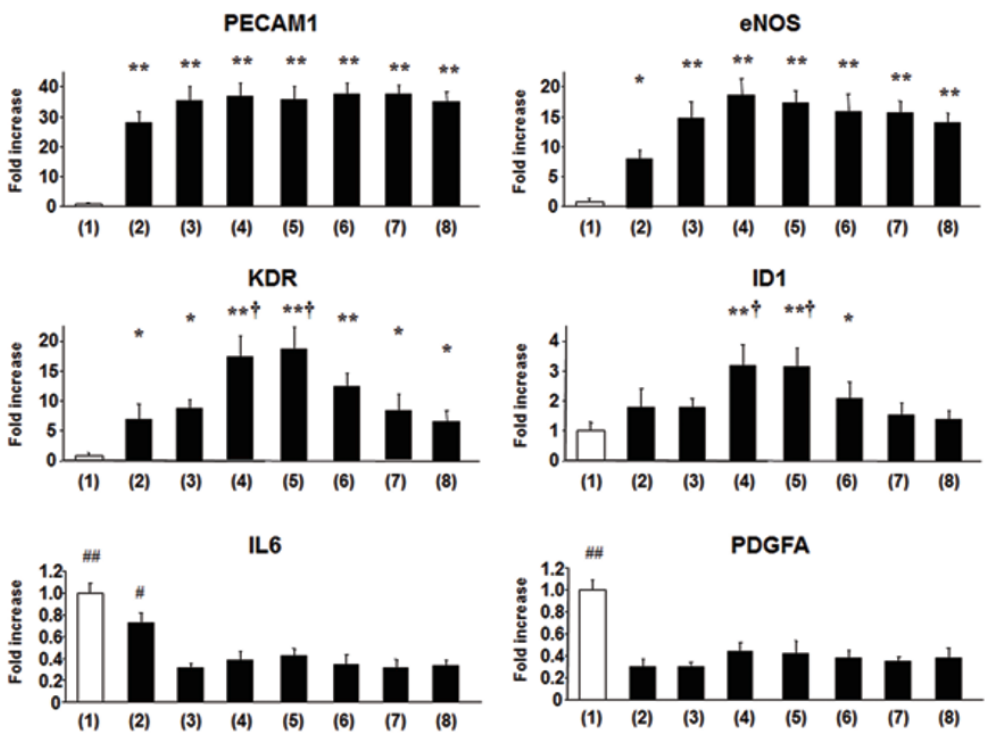
CD14 $(8.8 \% \pm 1.8 \%)$ or CD14 ${ }^{+}$ECs $(15.4 \% \pm 3.3 \%)$ (Figure 3B). The average number of cell progeny derived from single-plated $\mathrm{CD} 34^{+} \mathrm{CD} 14^{-} \mathrm{ECs}$ was 10 - to 30 fold greater than that from single-plated $\mathrm{CD} 34^{-} \mathrm{CD} 14^{-}$or CD $14^{+}$ECs (Figure 3C and 3D). Approximately half of the $\mathrm{CD} 34^{+} \mathrm{CD} 14^{-} \mathrm{ECs}$ sorted on day 6 became negative for CD34 on day 14 and exhibited weaker expression of KDR when cultured with VEGF-A in phase 3 (Figure $3 \mathrm{E})$, presumably indicating EC maturation.

Finally, to examine what percentage of cells would be putative hemogenic ECs, which are considered as intermediately differentiated vascular ECs that generate hematopoietic cells during embryogenesis [38, 39], we analyzed the expression ratio of c-kit, which is considered as one of the important markers of hemogenic ECs and their progeny hematopoietic progenitor cells as well as endothelial markers [38, 39], in each EC subpopulation. We found that the $\mathrm{CD} 14^{+}$EC subpopulation contained higher number of c-kit-positive cells $(20.4 \% \pm 3.3 \%)$, while the $\mathrm{CD} 34^{+} \mathrm{CD} 14^{-}$EC subpopulation contained much fewer number of c-kit-positive cells $(0.9 \% \pm 0.3 \%)$ (Supplementary information, Figure S4A-S4C), suggesting that the coexistence of hemogenic ECs/hematopoietic progenitor cells among the $\mathrm{CD} 34^{+} \mathrm{CD} 14^{-} \mathrm{EC}$ subpopulation was a rare event. Collectively, these data support the notion that hESC-derived $\mathrm{VEC}^{+} \mathrm{CD} 31^{+} \mathrm{CD} 34^{+} \mathrm{CD} 14^{-}$cells contain a discriminative EP population, and thereafter, we named this population as human PSC-derived EP.

The Notch signaling pathway inhibitor DAPT promotes $E P$ proliferation and blocks its differentiation into mature EC

Next, we sought to determine whether Notch signaling affects the differentiation and/or proliferation of $\mathrm{KDR}^{+}$precursors and EPs. First, we cultured the
$\mathrm{VEC}^{+} \mathrm{CD} 31^{+} \mathrm{CD} 34^{+} \mathrm{CD} 14^{-}$EPs sorted on day 6 with VEGF-A and recombinant human Dll4 (rhDll4) protein (a ligand of the Notch receptor) or the Notch signaling inhibitor DAPT in phase 3. The biological activity of rhDll4 and DAPT was evaluated by monitoring levels of active Notch1 (the Notch1 intracellular domain [N1ICD]) in nuclear extracts of cultured cells by immunoblotting (Figure $3 \mathrm{~F}$ and $3 \mathrm{G}$ ). Interestingly, combined treatment with VEGF-A and DAPT in phase 3 maintained a higher ratio of EPs $(84 \%)$ among the cultured endothelial lineage cells on day 14 compared to treatment with VEGFA alone (46\%), whereas VEGF-A and rhDll4 decreased the ratio of EPs $(28 \%)(P<0.01)$ (Figure $3 \mathrm{H})$. Similarly, combined treatment with VEGF-A and DAPT in phase 2 enhanced the induction of $\mathrm{VEC}^{+} \mathrm{CD} 31^{+} \mathrm{ECs}$, specifically CD $34^{+} \mathrm{CD} 14^{-}$EPs, whereas VEGF-A and rhD114 decreased ratios of both $\mathrm{VEC}^{+} \mathrm{CD} 31^{+}$ECs and CD $34^{+} \mathrm{CD} 14^{-}$EPs among ECs (Figure 3I).

To gain insights into the molecular basis of how inhibition of Notch signaling promotes endothelial differentiation, we monitored VEC and KDR expression after administration of VEGF-A and/or DAPT during phase 2 of differentiation. DAPT significantly increased the ratio of $\mathrm{KDR}^{+}$cells on day 6 , although treatment with DAPT alone only resulted in a few scattered $\mathrm{VEC}^{+}$cells (Supplementary information, Figure S5A). VEGF-A treatment in phase 2, after the priming with BMP4/GSK$3 \beta \mathrm{I}$, resulted in around $15 \% \mathrm{VEC}^{+}$of the total number of cells, but the total $\mathrm{KDR}^{+}$cell ratio was lower in cultures treated with VEGF-A alone than with DAPT alone. Interestingly, the combined use of DAPT and VEGF-A in phase 2 resulted in a substantially higher ratio of $\mathrm{VEC}^{+}$ cells, approaching 50\%, along with a higher ratio of total $\mathrm{KDR}^{+}$cells (Supplementary information, Figure S5A). Taken together, simultaneous treatment with DAPT and

Figure 2 Subpopulation analyses of $\mathrm{VEC}^{+} \mathrm{CD} 31^{+} \mathrm{ECs}$ for identifying an endothelial progenitor population. (A) Expression of KDR and CD34 in the VEC $C^{+} C D 31^{+}$ECs on day 6 was evaluated by FACS. (B) VEC ${ }^{+} \mathrm{CD} 31^{+}$ECs on day 6 were divided into three subpopulations (CD34 ${ }^{+} \mathrm{CD} 14^{-}, \mathrm{CD} 34^{-} \mathrm{CD} 14^{-}$, and $\left.\mathrm{CD} 14^{+}\right)$for further characterization. (C) CD14 mRNA expression in $\mathrm{CD}_{14}^{+}$and $\mathrm{CD} 14^{-}$populations sorted by FACS was analyzed with RT-PCR. (D) In vitro tube formation on Matrigel. Left, brightfield; right, EGFP. Scale bars, $100 \mu \mathrm{m}$. (E) High magnification photographs of tube formation in D (CD34 ${ }^{+}$CD14 ECs). Arrows indicate the lumens of the tubes formed by implanted CD $34^{+}$CD $14^{-}$ECs. Top, brightfield; bottom, EGFP. Scale bars, $50 \mu \mathrm{m}$. (F) Quantitative results of total tube area in the tube formation assay. HUCBC, human umbilical cord blood cell; HUVEC, human umbilical vein endothelial cell. ${ }^{*} P<0.01$. (G) The cell proliferation (MTS) assay showing the significant difference among the three subpopulations. $* P<0.01$. (H) Generated VEC ${ }^{+}$CD $31^{+}$EC numbers on day 14 derived from $1 \times 10^{4}$ cells of each EC subpopulation sorted on day $6 .{ }^{*} P<0.01$. (I) Molecular profiles of the three EC subpopulations on day 6 , non-EC (VEC $\left.{ }^{-} C D 31^{-}\right), H U C B C, H U V E C$, and CD34 ${ }^{+}$CD14- EC (EP)-derived cell 30 and 60 days after initial FACS sorting through several passages were analyzed using PCR arrays (SABiosciences), focusing on endothelial lineage-related genes. The clustergram image of array data shows differentially expressed genes among the populations. Red indicates increased expression, whereas green indicates decreased expression. (J) Quantitative comparisons of mRNA expression of the selected genes in the PCR array data in I among the populations. (1) non-EC (VEC $\left.{ }^{-} C D 31^{-}\right)$; (2) CD14 ${ }^{+}$EC; (3) CD34 ${ }^{-}$CD14 ${ }^{-}$EC; (4) $\mathrm{CD}^{+}{ }^{+} \mathrm{CD} 14^{-} \mathrm{EC}(\mathrm{EP})$; (5) HUCBC; (6) HUVEC; (7 and 8) EP-derived cell 30 days and 60 days after sorting. $* P<0.05$ and $* * P<0.01$ vs non-ECs. $\uparrow P<0.01$ vs (2) and (3). $\# P<0.01$ and $\# \# P<0.0001$ vs (3) and (4). Error bars, SD $(n \geq 3)$. 
VEGF-A resulted in a synergistic effect, suggesting that DAPT might enhance responsiveness of hESC-derived mesodermal precursors to VEGF-A for endothelial differentiation through maintaining or activating KDR activity. This notion is supported by a growing body of evidence showing that VEGF-A/KDR signaling controls expression of the Notch ligand Dll4 in ECs as part of a negative feedback loop, where Dll4-mediated activation of Notch receptors results in repression of KDR transcription [40-42]. Quantitative RT-PCR and immunoblot- ting confirmed elevated levels of D114 mRNA and protein in differentiating hESCs treated with VEGF-A (Supplementary information, Figure S5B and S5C) along with upregulation of the canonical Notch downstream target genes Hes1, Hey1, and Hey2 (Supplementary information, Figure S5D-S5F). Notably, administration of DAPT together with VEGF-A did not affect the expression level of Dll4 compared to VEGF-A treatment alone, but significantly attenuated VEGF-A-induced upregulation of Notch target gene expression (Supplementary informa-
A

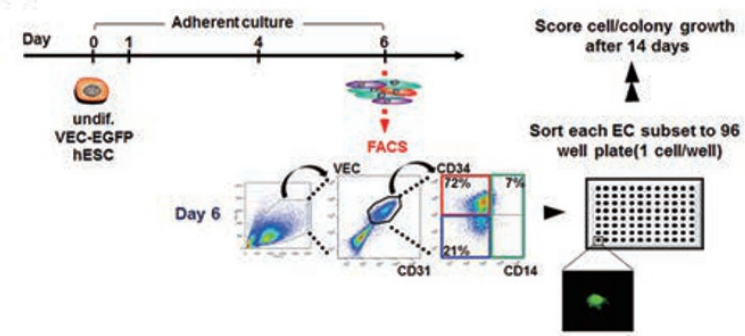

D

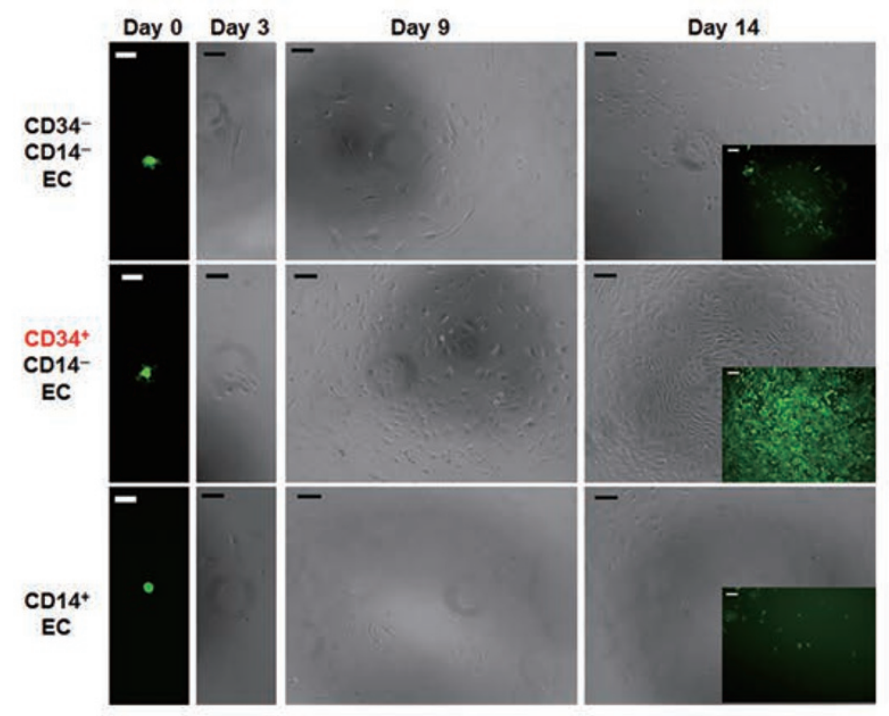

G
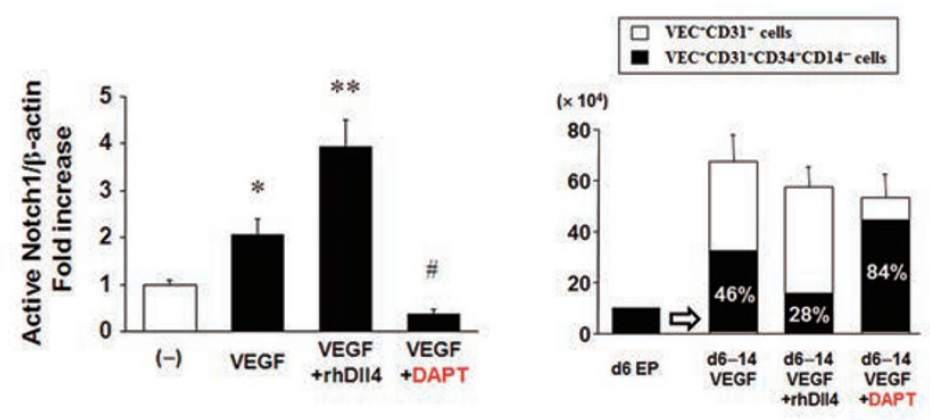

B

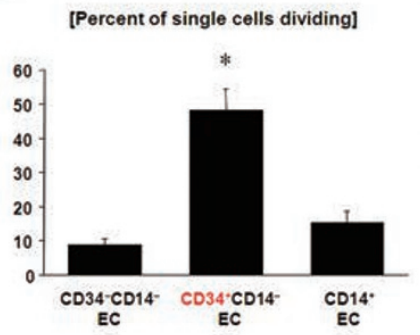

C

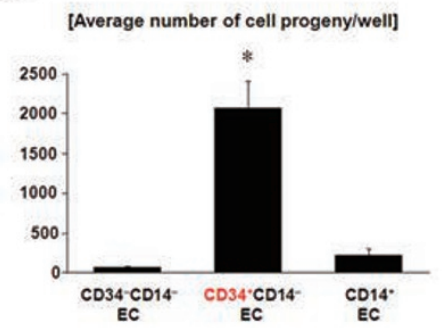

E

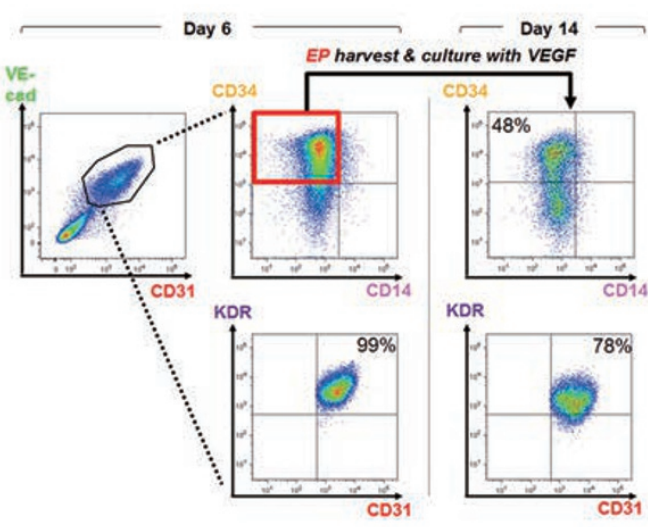

$\mathbf{F}$

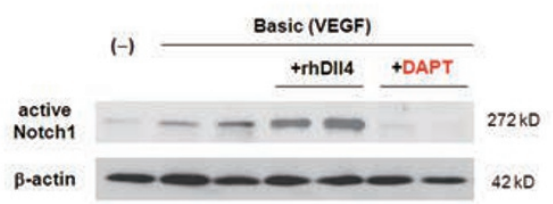

I

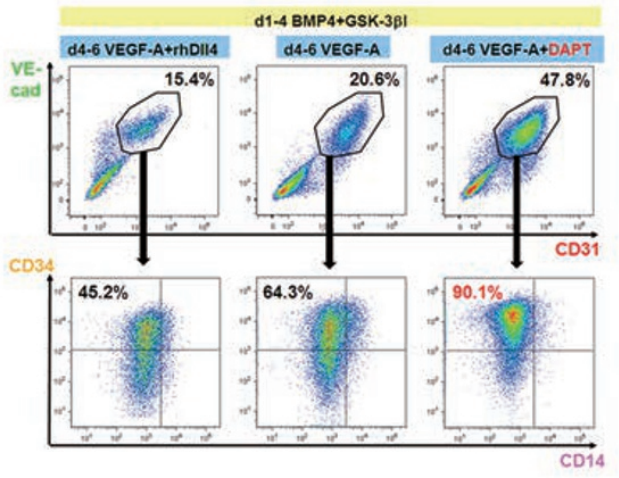


tion, Figure S5B-S5F).

Inhibition of Notch signaling in combination with VEGFA blocks $K D R^{+}$mesodermal precursor differentiation into non-endothelial lineages

$\mathrm{KDR}^{+}$cells have previously been described as multipotent precursors during mesodermal development, with a potential to differentiate into not only ECs but also vascular SMCs and cardiomyocytes [24, 25, 40]. To investigate the direct effects of DAPT on the developmental potential of $\mathrm{KDR}^{+}$precursors, we sorted hESC-derived $\mathrm{KDR}^{+}$cells on day 4 and cultured them under differentiating conditions with rhDll4 or DAPT (Figure 4A). After 4 days, VEGF-A treatment promoted endothelial differentiation of $\mathrm{KDR}^{+}$precursors and decreased KDR expression, along with limited but detectable differentiation into vascular SMCs (PDGFR $\alpha$ - or smooth muscle myosin heavy chain (SM-MHC)-positive) and cardiomyocytes (cTnT-positive) (Figure 4A-4C). Combined treatment with rh-Dll4 and VEGF-A enhanced differentiation of $\mathrm{KDR}^{+}$precursors into vascular SMC and cardiomyocyte lineages, and attenuated endothelial differentiation (Figure $4 \mathrm{~A}-4 \mathrm{C}$ ), which is in keeping with previous reports $[43,44]$. In contrast, treatment with DAPT and VEGF-A significantly enhanced the induction of ECs, with maintained KDR expression, while attenuating differentiation into SMCs and cardiomyocytes (Figure 4A-4C).

To investigate whether the findings obtained in the hESC-based endothelial differentiation system also would be applicable to human fetal heart-derived $\mathrm{KDR}^{+}$ precursors, we collected the cardiac mesenchymal cell (CMC) fractions, including $\mathrm{KDR}^{+}$precursors, derived from human fetal hearts ( 9 weeks of gestation), as previ- ously described [45]. Four to 6 days after culture with or without DAPT, FACS analyses, and immunocytochemical studies revealed that DAPT treatment significantly increased the $\mathrm{KDR}^{+}$cell ratio/number compared to control (Figure 4D and 4E). These findings suggest that DAPT promotes proliferation and inhibits differentiation of $e x$ vivo-cultured $\mathrm{KDR}^{+}$precursor cells from human fetal heart tissue. It also further corroborates the notion that DAPT treatment with VEGF-A induces and maintains $\mathrm{EP}\left(\mathrm{VEC}^{+} \mathrm{CD} 31^{+} \mathrm{CD} 34^{+} \mathrm{CD} 14^{-} \mathrm{KDR}^{\text {high }}\right)$ identity during hPSC endothelial differentiation through a two-pronged mechanism: VEGF-A/DAPT treatment instructs $\mathrm{KDR}^{+}$ precursors to adopt the endothelial fate at the expense of non-endothelial lineages, and inhibits the negative VEGF-Notch feedback mechanism resulting in KDR downregulation and further maturation along the endothelial lineage.

\section{The canonical Notch signaling pathway attenuates KDR} expression in EPs

Our studies showed that VEGF-A alone in phase 2 induced differentiation of hESC-derived $\mathrm{KDR}^{+}$precursors to the endothelial lineage, but also attenuated levels of KDR expression (Figure 4A), resulting in a lower ratio of $\mathrm{VEC}^{+} \mathrm{CD} 31^{+} \mathrm{CD} 34^{+} \mathrm{CD} 14^{-} \mathrm{KDR}^{\text {high }}$ EPs among $\mathrm{VEC}^{+} \mathrm{CD} 31^{+}$ECs (Figure 3I). DAPT attenuated the VEGF-A-induced upregulation of Notch target gene expression (Supplementary information, Figure S5D-S5F), leading us to hypothesize that Notch signaling might mediate the effect of VEGF-A on KDR expression, and that DAPT might block the decrease of KDR expression through the downregulation of the Notch downstream targets.

Figure 3 The $\mathrm{CD} 34^{+} \mathrm{CD} 14^{-}$subpopulation among $\mathrm{VEC}{ }^{+} \mathrm{CD} 31^{+} \mathrm{ECs}$ represents an endothelial progenitor population, and Notch signaling controls their proliferation or further maturation. (A) Schematic of the single-cell culture experiment. On day 6 in the endothelial differentiation, the single cells of the three subpopulations (CD34 ${ }^{+}$CD $\left.14^{-}, \mathrm{CD}^{-} 4^{-} \mathrm{CD} 14^{-}, \mathrm{CD}^{+} 4^{+}\right)$among hESC-derived VEC ${ }^{+} \mathrm{CD} 31^{+}$cells were sorted onto 96 well plates (1 cell/well) and cultured under endothelial cell conditions for 14 days. $(\mathbf{B}, \mathbf{C})$ Percent of single cells dividing $(\mathbf{B})$, defined as undergoing $\geq 1$ cell division, and the average number of

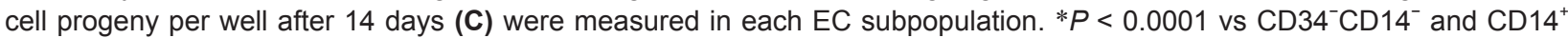
EC. (D) Representative photomicrographs of the single CD $34^{-} \mathrm{CD} 14^{-}$(top), $\mathrm{CD} 34^{+} \mathrm{CD} 14^{-}$(middle), and CD14 ${ }^{+} \mathrm{EC}$ (bottom) culture assay. Scale bars, $50 \mu \mathrm{m}$ (day 0) and $100 \mu \mathrm{m}$ (day 3, day 9 and day 14). Day 3, day 9 and day 14, brightfield; day 0 and insets of day 14, VEC-EGFP. (E) Expression of CD34 and KDR on day 14 in the cultured cells derived from the EPs $\left(\mathrm{VEC}^{+} \mathrm{CD} 31^{+} \mathrm{CD} 34^{+} \mathrm{CD} 14^{-}\right)$sorted on day 6 . After sorting, EPs were cultured with VEGF-A and analyzed with FACS on day 14. (F) Representative images of western blot analysis for active Notch1 (the Notch1 intracellular domain (N1ICD)) in nuclear extracts of the cultured cells treated with VEGF-A with or without rhDII4 or DAPT. (G) Quantitative results in F are shown. Bands were scanned and quantified with Image $J$. The signal intensity was normalized to $\beta$-actin expression. (-) indicates no molecules. ${ }^{*} P<0.05$ vs $(-),{ }^{* *} P<0.01$ vs $(-)$ and VEGF-A, $\# P<0.01$ vs VEGF-A, and VEGF-A + rhDII4. (H) Diverse ratios of $\mathrm{VEC}^{+} \mathrm{CD} 31^{+} \mathrm{CD} 34^{+} \mathrm{CD} 14^{-}$cells among VEC ${ }^{+} \mathrm{CD} 31^{+}$cells on day $14.1 \times 10^{4} \mathrm{VEC}^{+} \mathrm{CD} 31^{+} \mathrm{CD} 34^{+} \mathrm{CD} 14^{-}$cells (EPs) were sorted on day 6 and cultured with VEGF-A with or without rhDII4 or DAPT in phase 3. Notably, combined treatment with VEGF$\mathrm{A}$ and DAPT maintained a higher ratio of $\mathrm{VEC}{ }^{+} \mathrm{CD} 31^{+} \mathrm{CD} 34^{+} \mathrm{CD} 14^{-}$cells among the cultured cells on day 14 , whereas VEGFA and rhDll4 decreased that ratio $(P<0.01)$. (I) The differential EC $\left(\mathrm{VEC}^{+} \mathrm{CD} 31^{+}\right)$and EP $\left(\mathrm{CD} 34^{+} \mathrm{CD} 14^{-} \mathrm{EC}\right)$ ratios on day 6 induced with treatment with VEGF-A with or without rhDll4 or DAPT in phase 2. Error bars, SD $(n \geq 3)$. 
A

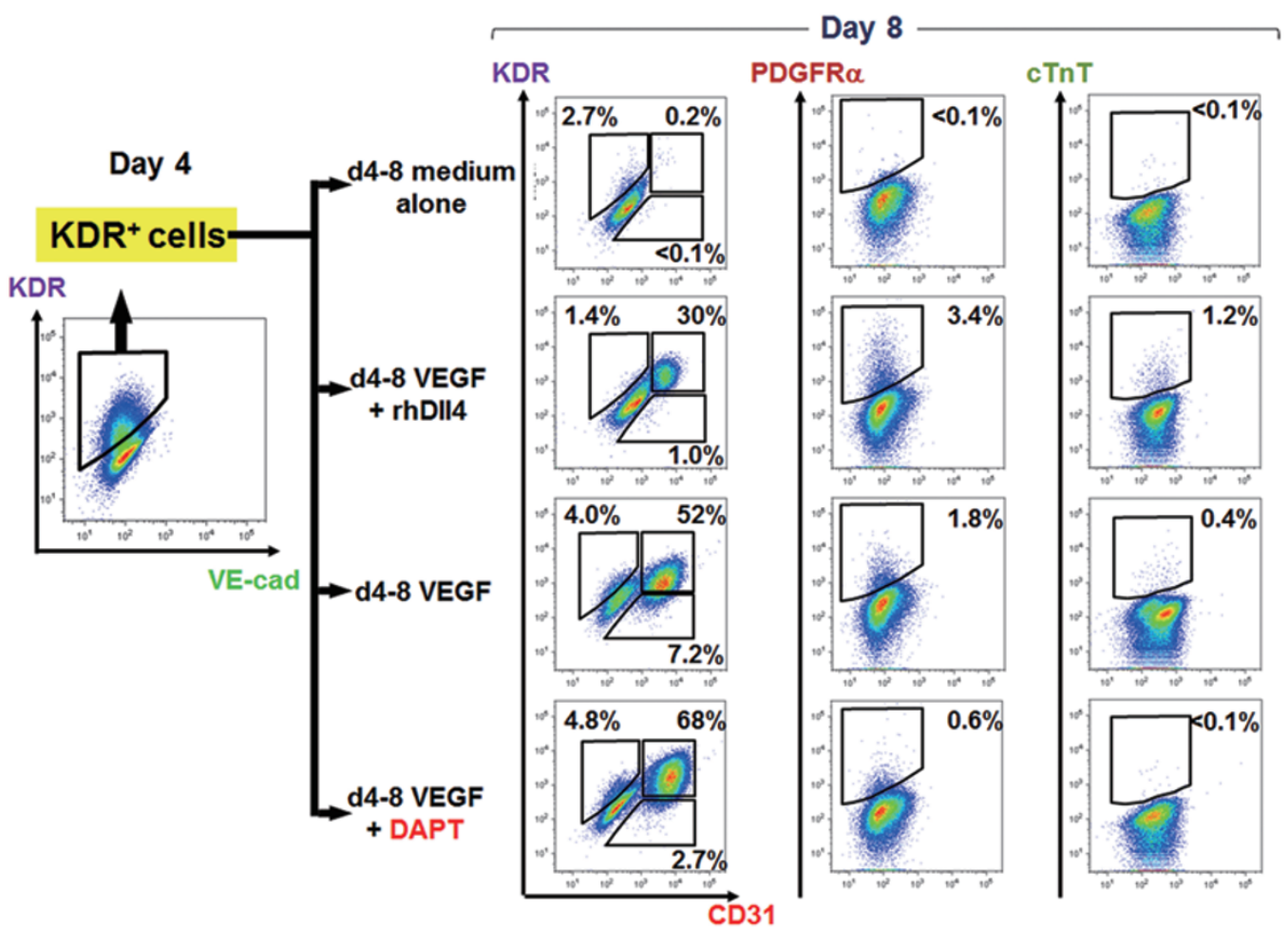

B
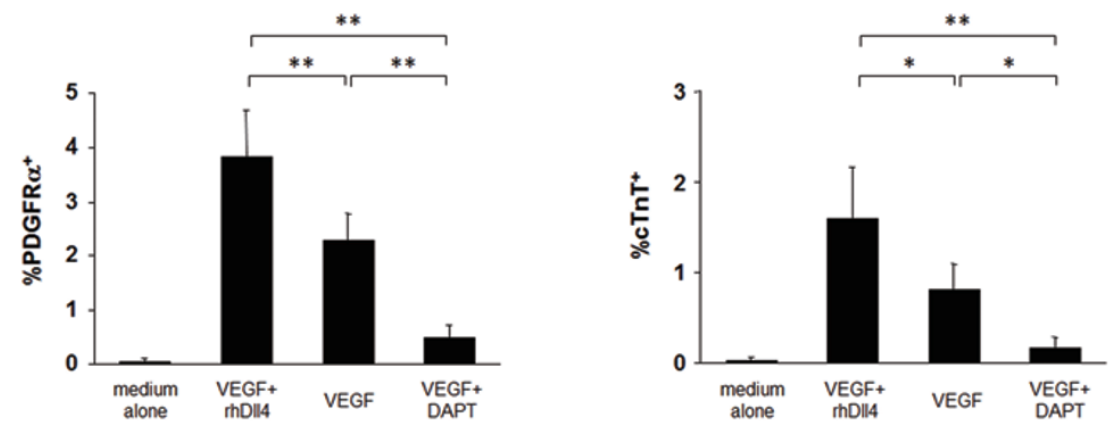

C
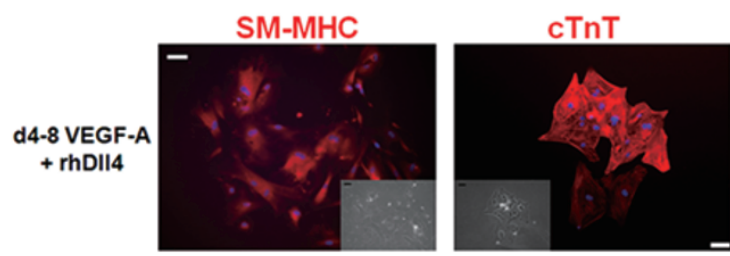

D
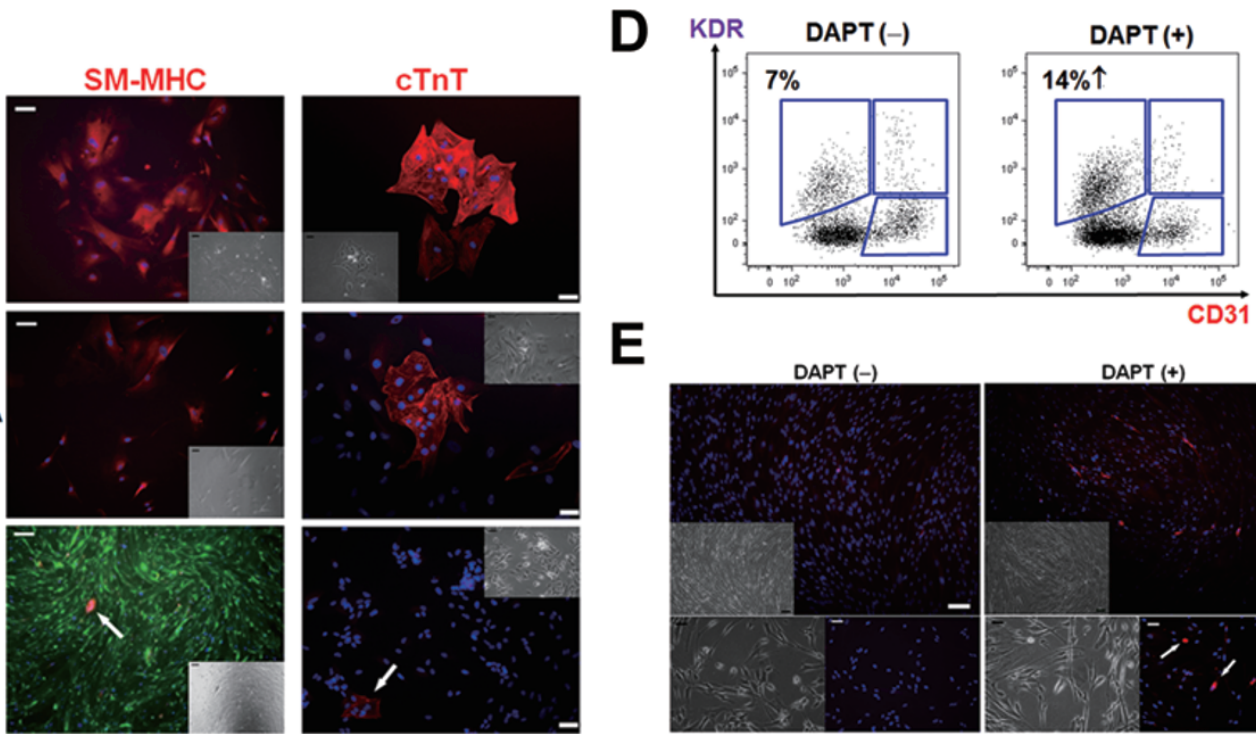

E

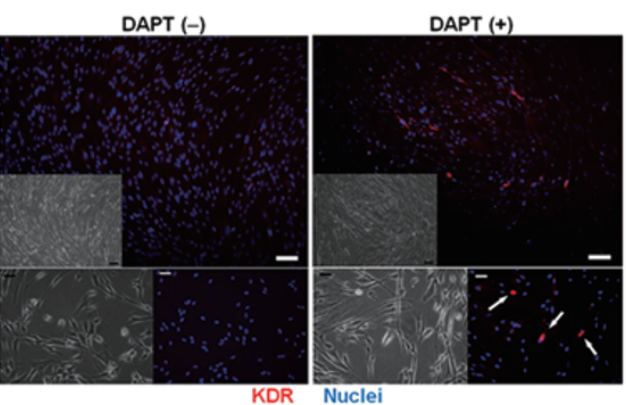


To rigorously test this hypothesis and to gain further mechanistic insights into how Notch signaling regulates maturation of EPs highly expressing KDR, we generated pKDR-Luc, a KDR reporter vector where a human KDR promoter fragment $(-900,+296)[23]$ controls expression of the firefly luciferase gene (Figure 5A). hESCs were transfected with pKDR-Luc in the undifferentiated state, and $6 \mathrm{~h}$ after transfection, differentiation was initiated through treatment with BMP4 and/or GSK-3 3 I for $12 \mathrm{~h}$ (Figure 5B). We found that BMP4 treatment increased luciferase activity by fourfold compared with control (Figure 5C). Although GSK-3ßI alone increased the KDR-luciferase activity only modestly, the combined administration of BMP4 and GSK-3 $\beta$ I increased luciferase activity by eightfold (Figure $5 \mathrm{C}$ ). We then treated the cells with VEGF-A, rhDll4, and/or DAPT for additional $18 \mathrm{~h}$ (Figure 5B). VEGF-A attenuated the BMP4-induced increase of KDR-reporter activity and in addition, rhDll4 (with VEGF-A) further decreased luciferase activity (Figure 5C). Interestingly, DAPT blocked the VEGF-Ainduced decrease of KDR-reporter activity, maintaining luciferase activity at a high level (Figure 5C).

DAPT has been shown to function as a specific inhibitor of $\gamma$-secretase [46], an enzymatic multi-subunit complex catalyzing the liberation of the membrane-tethered NICD, the final step in ligand-mediated Notch receptor activation. Because Notch is not the only substrate of $\gamma$-secretase, we performed knockdown studies of core components of the Notch signaling pathway to validate that the effect of DAPT is due to its effects on Notch signaling. Tetracycline-inducible shRNA lentiviral vectors, targeting Notch1, the DNA-binding protein RBP-Jא that targets nuclear NICD to specific enhancers and promoters of downstream target genes [47], and Notch signaling downstream targets Hes1 and Hey1 [48] were generated, and the corresponding virus particles were transduced into undifferentiated hESCs (Supplementary information, Figure S6A). First, we confirmed the tetracycline-inducible knockdown effects for the expression of Notch1,
RBP-J $\kappa$, Hes1, and Hey1, respectively, by quantitative RT-PCR measured at 12-24 h after doxycycline $(1 \mu \mathrm{g} /$ $\mathrm{ml}$ ) administration (Supplementary information, Figure S6B). The inducible shRNA hESC lines were then differentiated into endothelial lineage cells using the same protocol with or without doxycycline treatment from day 4 (Figure 5D). As shown in Figure 5E and 5F, knockdown of Notch1 or RBP-JK induced by doxycycline in phase 2 significantly promoted endothelial differentiation with the production of a higher number of $\mathrm{CD} 34^{+} \mathrm{CD} 14^{-} \mathrm{EPs}$, compared with the case without doxycycline or control (non-targeting) shRNA-transduced hESCs. Similarly, genetic knockdown of Hes1 or Hey1 also promoted generation of EPs, although the effects were more modest compared to the effects of Notch1 and RBP-JK knockdown (Figure 5G). Next, the undifferentiated inducible shRNA hESC lines were transfected with pKDR-Luc and after $6 \mathrm{~h}$ stimulated with BMP4 for the next $12 \mathrm{~h}$. We then treated the cells with VEGF-A in the presence or absence of doxycycline $(1 \mu \mathrm{g} / \mathrm{ml})$ for additional $18 \mathrm{~h}$ (Figure $5 \mathrm{H})$. Thirty-six h after pKDR-Luc transfection, VEGF-A alone decreased the BMP4-induced KDR-luciferase activity, whereas knockdown of Notch1 or RBP-J $\kappa$ blocked the VEGF-A-mediated decrease of KDR-reporter activity (Figure 5I). Furthermore, knockdown of Hes1 or Hey 1 also partially blocked the VEGF-A-mediated decrease of the KDR-luciferase activity (Figure 5I). These data strongly support the notion that treatment with VEGF-A in combination with DAPT or genetic knockdown of core components of the Notch signaling pathway in phase 2 promotes $\mathrm{hESC}$ differentiation into $\mathrm{CD} 34^{+} \mathrm{CD} 14^{-} \mathrm{EPs}$ by maintaining KDR promoter activity via downregulation of the Notch downstream targets.

Transplanted hPSC-derived EPs form functional capillary networks and anastomose with host vessels in vivo and improve heart function in mice after MI

Our finalized strategy for the production of hPSCderived EPs $\left(\mathrm{VEC}^{+} \mathrm{CD} 31^{+} \mathrm{CD} 34^{+} \mathrm{CD} 14^{-}\right)$is illustrated

Figure 4 DAPT enhances proliferation rather than differentiation of the $\mathrm{KDR}^{+}$precursors. (A) Differentiation of $\mathrm{KDR}^{+}$mesodermal precursors into the three cardiovascular lineages: endothelial cells $\left(\mathrm{CD} 31^{+}\right)$, smooth muscle cells $\left(\mathrm{PDGFR} \alpha^{+}\right)$and cardiomyocytes $\left(\mathrm{cTnT}^{+}\right)$. $\mathrm{KDR}^{+}$precursors were harvested on day 4 and cultured with VEGF-A with or without rhDII4 or DAPT up to day 8 prior to FACS analyses. (B) Quantitative results of the PDGFR $\alpha$ - and CTnT-positive ratios at day 8 in each KDR ${ }^{+}$derived population, differentially treated as in $\mathbf{A}$. $* P<0.05$ and $* * P<0.01$. Error bars, SD $(n=5)$. (C) Immunocytochemical images of $\mathrm{KDR}^{+}$precursor-derived smooth muscle cells (left) and cardiomyocytes (right). SM-MHC and cTnT signals are indicated as red. DAPT attenuated VEGF-A-induced differentiation of $\mathrm{KDR}^{+}$precursors into smooth muscle cells and cardiomyocytes (indicated as arrows, respectively). Green, VEC-EGFP; blue, nuclei. Insets, brightfield. Scale bars, $50 \mu \mathrm{m}$ (cTnT) and $100 \mu \mathrm{m}$ (SM-MHC). (D) Expression of KDR in the human fetal heart (9 weeks of gestation)-derived cardiac mesenchymal cell fractions, treated with or without DAPT, was evaluated with FACS. (E) Immunocytochemical images of $\mathrm{KDR}^{+}$precursors in the human fetal heart-derived cardiac mesenchymal cell fractions. DAPT increased the number of KDR ${ }^{+}$precursors (red, arrows). Blue, nuclei; top insets and bottom left, brightfield. Scale bars, $50 \mu \mathrm{m}$ (bottom) and $100 \mu \mathrm{m}$ (top). 
A

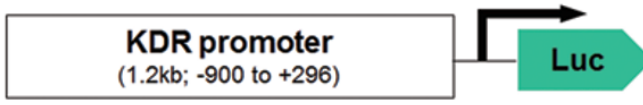

B

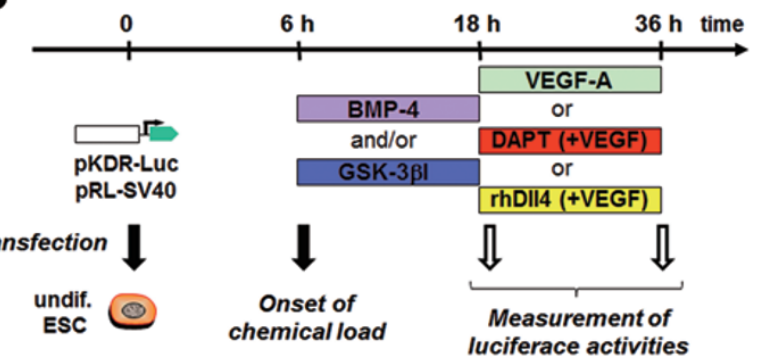

C

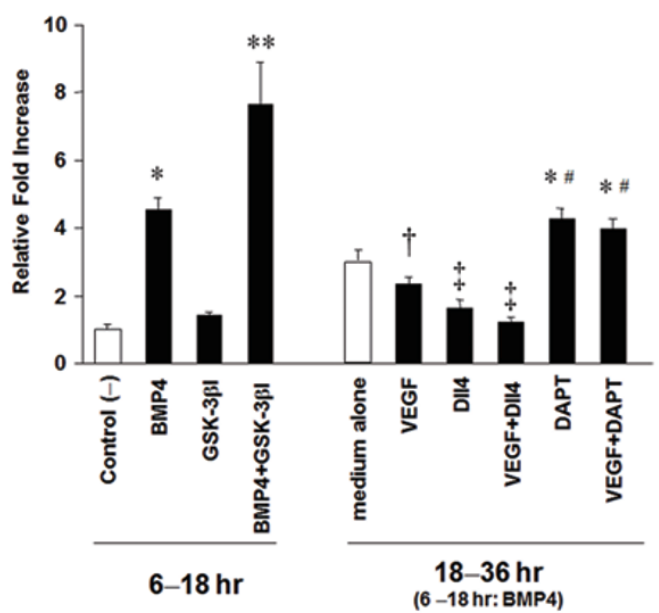

D

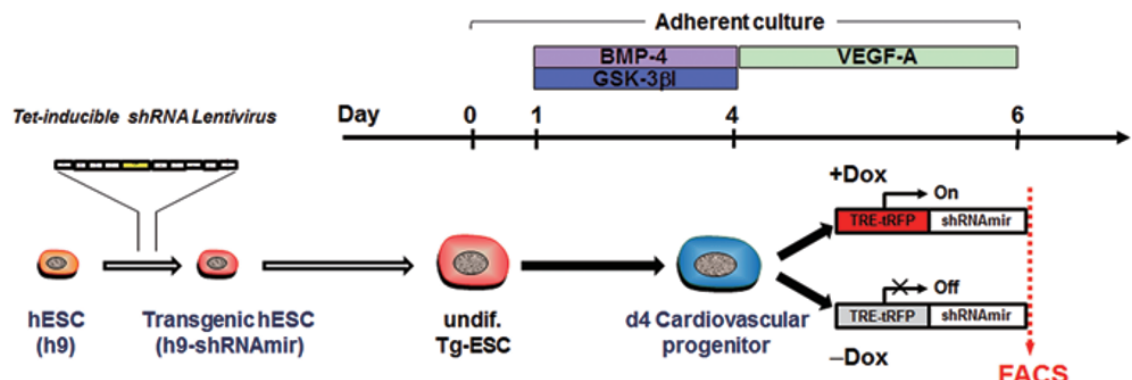

E
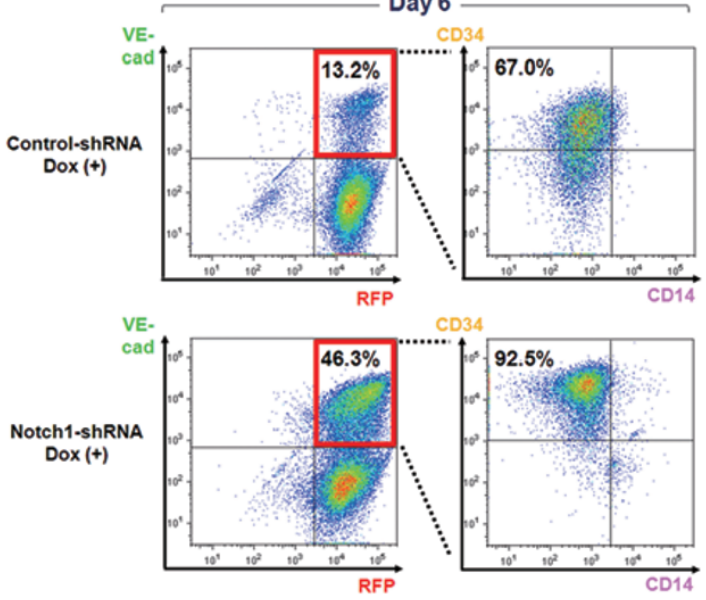

H

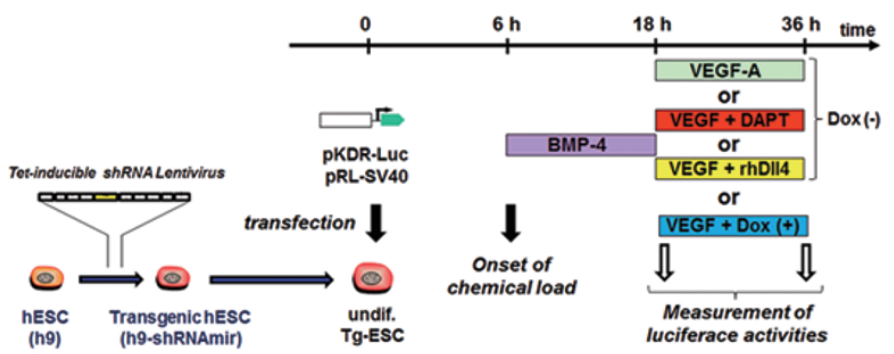

$\mathbf{F}$
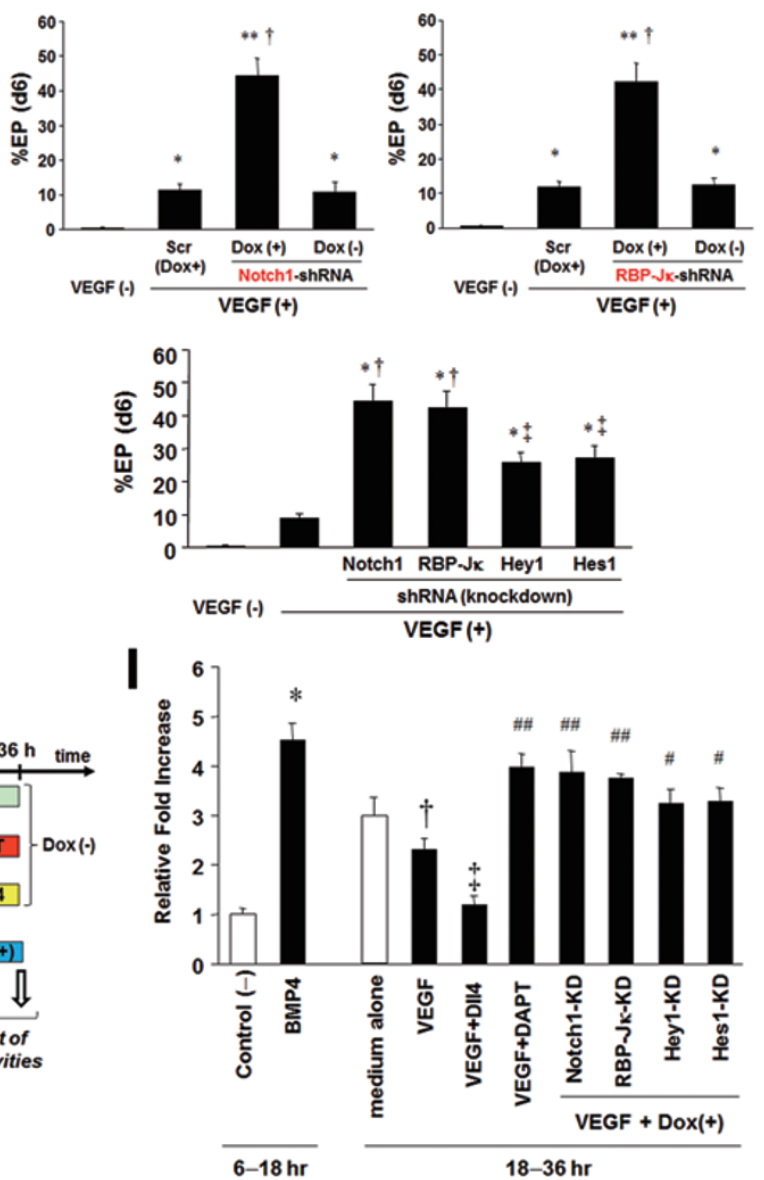
in Figure 6A and consists of a stepwise combination of treatment with: (1) BMP4 and GSK-3 $\beta$ I in phase 1 (day 1-4); and (2) DAPT and VEGF-A with PLGF and HGF in phase 2 (day 4-6), using method B described above. With this simple approach, 2.5 million of EPs could be generated in only 6 days from 1 million of hPSCs (Figure $6 \mathrm{~B}$ ). When the 2.5 million of EPs were cultured with VEGF-A in phase 3, 20 million $\mathrm{VEC}^{+} \mathrm{CD} 31^{+}$ECs were generated on day $14(\mathrm{hPSC} / \mathrm{EC}$ ratio $=1: 20)($ Figure $6 \mathrm{~B})$. To test the vasculo/angiogenic effects in vivo, the hPSCderived EPs ( $\geq 1$ million) were directly transplanted with Matrigel plugs subcutaneously into non-obese diabetic/ severe combined immunodeficiency (NOD/SCID) mice. Two weeks later, the transplanted EPs formed capillary vessel networks in Matrigel plugs (Figure 6C), and those neo-vessels formed by transplanted VEC-EGFP ${ }^{+}$ EPs exhibited functional anastomosis with host vessels of NOD/SCID mice (Figure 6D and 6E). This finding was supported by the evidence that VEC-EGFP ${ }^{+}$EPs colocalized with lectin ${ }^{+}$cells (Figure $6 \mathrm{~F}$ ). The transplanted EP-derived neo-vessels continued to function for more than 3 months (Figure 6G). Quantification of the area of newly formed vessels revealed a correlation with the number of transplanted EPs (Figure 6H). Furthermore, a direct comparison between $\mathrm{VEC}^{+} \mathrm{CD} 31^{+} \mathrm{CD} 34^{+} \mathrm{CD} 14^{-}$ EPs harvested on day 6 of hPSC differentiation and $\mathrm{VEC}^{+} \mathrm{CD} 31^{+}$ECs harvested on day 14 of hPSC differentiation or HUVECs revealed that grafted EPs exhibited an increased (three to fivefold) capacity to form vascular networks in vivo after grafting, compared to $\mathrm{VEC}^{+} \mathrm{CD} 31^{+}$ ECs or HUVECs (Figure 6H). We did not see any tumor formation after transplantation of hPSC-derived EPs/ ECs.

Finally, to examine whether hPSC-derived EPs have the efficacy to promote vasculo/angiogenesis and to improve the organ function in a mouse diseased model such as MI, we injected $1 \times 10^{7}$ EPs directly into ischemic (peri-infarct) regions of myocardium immediately after ligation of the left anterior descending coronary artery (LAD). Twenty-eight days after MI, the transplanted EPs significantly attenuated left ventricle (LV) remodeling, as determined by echocardiography measurement of LV end-diastolic diameter (LVEDD) and LV end-systolic diameter (LVESD), and thereby improved LV function, as determined by echocardiography measurement of fractional shortening (FS) (Supplementary information, Figure S7A and S7B). In histology, the EPs directly engrafted into microcirculation in ischemic myocardium and increased capillary density, which was derived from both the incorporation of $\mathrm{EGFP}^{+}$EPs into native vessels and the increased number of native vessel ECs (Supplementary information, Figure S7C and S7D). We found that a subset of transplanted EP-derived vessels were covered with vascular SMCs (SM-MHC) ${ }^{+}$, which is consistent with mature vessels, in the peri-infarct regions of myocardium (Supplementary information, Figure S7E).

Figure 5 DAPT or genetic knockdown of the Notch signaling pathway maintains KDR promoter activity through downregulation of Notch signaling downstream targets. (A) A pKDR-Luc vector construct, containing the human KDR promoter fragment $(-900,+296)$ [23] upstream of the firefly luciferase gene. (B) Schematic of the dual-luciferase assay experiments. Undifferentiated hESCs were transfected with pKDR-Luc and pRL-SV40 (control) and after $6 \mathrm{~h}$, stimulated with BMP4 and/or GSK-3 $3 \mathrm{I}$ for the next $12 \mathrm{~h}$. Then, the cells were stimulated with VEGF-A, DAPT (with VEGF-A), or rhDIl4 (with VEGF-A). $18 \mathrm{~h}$ and $36 \mathrm{~h}$ after the transfection, dual luciferase activities were measured with a luminometer. (C) The relative fold induction of firefly luciferase activity normalized to control Renilla luciferase activity was determined. $* P<0.01 \mathrm{vs} \mathrm{control,} * * P<0.0001$ vs control, and $P<0.01$ vs BMP4, $\uparrow P<0.05$ vs BMP4, $\ddagger P<0.01$ vs BMP4, $\# P<0.01$ vs VEGF. Error bars, SD ( $n \geq 3)$. (D) Schematic of the endothelial differentiation experiments using the tetracycline (Tet)-inducible shRNA hESC lines. Undifferentiatied hESCs were initially transduced with Tet-inducible shRNA lentiviruses targeting a Notch signaling pathway (Notch1, RBP-JK, Hey1, and Hes1) and then differentiated into endothelial lineage cells using the same protocol with or without doxycycline treatment $(1 \mu \mathrm{g} / \mathrm{ml})$ from day 4. (E) Expression of RFP (a marker of cells expressing shRNA), VEC, CD34, and CD14 was evaluated with FACS on day 6 in endothelial differentiation of the Tet-inducible shRNA hESCs. Genetical knockdown of Notch1 induced by doxycycline (Dox) in phase 2 enhanced the production of both VEC ${ }^{+}$ECs and CD $34^{+} \mathrm{CD} 14^{-}$EPs among ECs compared to control (non-targeting shRNA-transduced hESCs). (F) Percent EP on day 6 in endothelial differentiation of the Tet-inducible shRNA hESCs. Genetic knockdown of Notch1 (left) or RBP-JK (right) promoted EP induction, compared to Scr (Scramble no-targeting ShRNA) and Dox (-). *P< 0.05 vs VEGF $(-), * * P<0.0001$ vs VEGF $(-), \dagger P<0.0001$ vs Scr and Dox (-). (G) Percent EP on day 6 of each Tet-inducible shRNA hESC line. Although the degree was modest, genetic knockdown of Hey1 or Hes 1 also promoted EP induction. ${ }^{*} P<0.0001$ vs. VEGF $(-), \dagger P<0.0001$ vs. VEGF $(+), \ddagger P<0.01$ vs. VEGF (+). (H) Schematic of the dual-luciferase assay experiments using the Tet-inducible shRNA hESCs. Tet-inducible shRNA hESC lines were transfected with pKDR-Luc and after $6 \mathrm{~h}$, stimulated with BMP4 for $12 \mathrm{~h}$, followed by the incubation with VEGF-A with or without Dox for additional $18 \mathrm{~h}$. (I) Results of the dual-luciferase assay using the Tet-inducible shRNA hESCs. Similarly to DAPT, genetic knockdown of Notch1 or RBP-JK could maintain KDR promoter activity at a high level. Similarly, Hey1 or Hes1 knockdown also resulted in higher luciferase levels than control shRNA. ${ }^{*} P<0.01$ vs control, $\dagger P<0.05$ vs BMP4, $₫ P<0.01$ vs BMP4, \#\# $P<0.01$ vs VEGF, $\# P<0.05$ vs VEGF. Error bars, SD $(n \geq 3)$. 
A
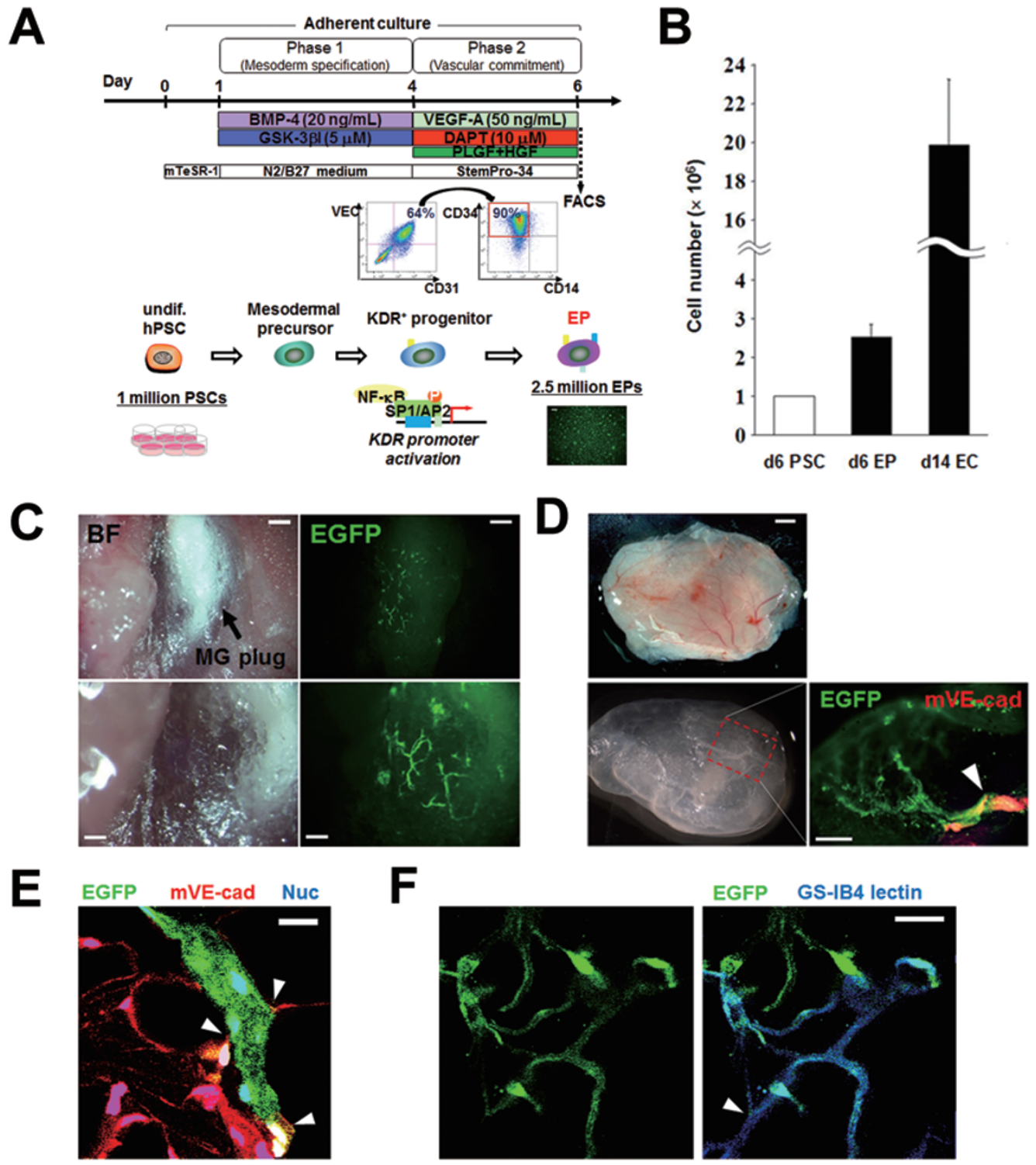

\section{$\mathbf{G}$}
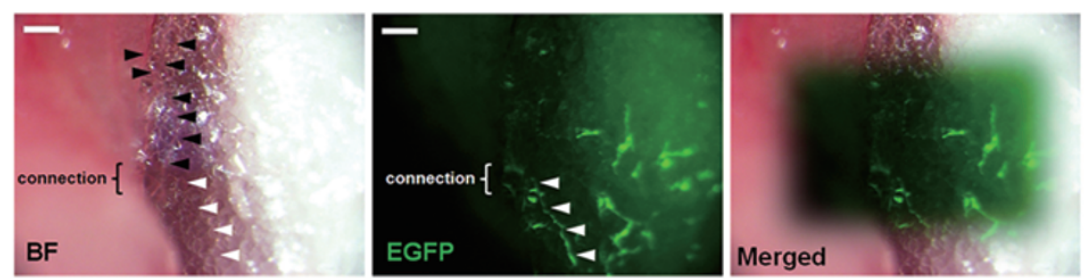

H

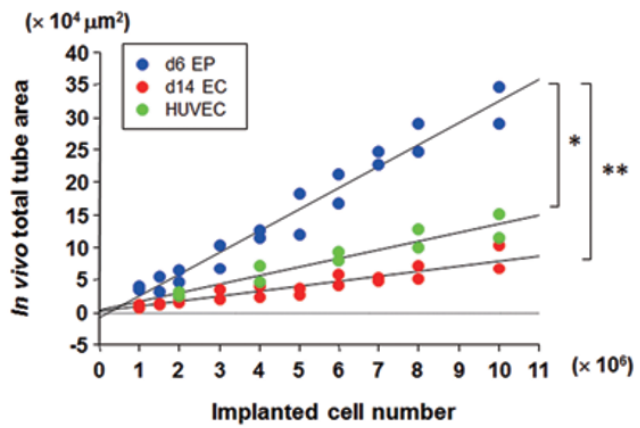


On the survival analysis up to day 45 after MI, the EPinjected mice group revealed the better survival rate (64.3\%), compared to control (vehicle) group (28.6\%), although not statistically significant $(n=14$, respectively; $P=0.09$ by log-rank test) (Supplementary information, Figure S7F).

These data provide direct experimental evidence that the EPs generated using the protocol described herein form functional vessel networks that anastomose with vessels of the host following transplantation with beneficial outcomes, even in the diseased model. Moreover, our results suggest that EPs may constitute a better cellular vehicle for revascularization efforts than mature ECs.

\section{Discussion}

Ischemic cardiovascular disease is the leading cause of death worldwide, despite recent progress in therapeutic treatment [49]. Regenerative cellular therapy is a novel promising approach for therapeutic revascularization in ischemic cardiovascular disease $[1,2]$. However, due to the limited regenerative capacity of mature vascular ECs $[2,5]$, generation of large numbers of vascular ECs will likely be required for sufficient contribution to the vasculature to have clinical impact. In this regard, EPs derived from bone marrow and peripheral blood mononuclear cells have been investigated as a candidate cell population to enhance ischemia-induced angio/vasculogenesis following ischemic cardiovascular disease [12-14]. However, the number of EPs existing in adult bone marrow and circulating blood is prohibitively low, and amplification of EPs is challenging [14, 28, 29]. In addition, the molecular signature of EPs has not been completely defined, except for partly accepted molecular marker specific to EPs [30, 31]. Thus, there have been few successful studies where large numbers of EP-derived ECs with the capacity to form functional vessels following grafting could be obtained [50-52].

hPSCs exhibit multi-lineage potential and the ability to self-renew indefinitely $[3,4]$, and constitute a promising source for the production of endothelial lineage cells, including EPs and ECs. However, protocols for robust differentiation of hPSCs into large numbers of functional endothelial lineage cells have been elusive [6-9], largely due to an incomplete understanding of the specific developmental stimuli controlling the specification of mesodermal precursors into endothelial lineage cells [17, 53, 54].

To date, the most successful approaches for directed differentiation of hPSCs into endothelial lineage cells (Supplementary information, Table S2) [6-9, 17, 18, $54,55]$ have relied on application of secreted extracellular factors, primarily BMP4 and VEGF-A. In addition, James et al. [17] have reported that the TGF $\beta$ inhibitor SB431542 enhanced EC differentiation of hESCs when administered in combination with VEGF-A during days 7-14 of differentiation, corresponding to endothelial commitment and expansion of differentiating cultures. However, in their report, administration of SB431542 resulted in modest efficiency for $\mathrm{CD} 31^{+} \mathrm{VEC}^{+} \mathrm{EC}$ formation, corresponding to only a small minority $(1.8 \%)$ of the total number of cells on day 14 of differentiation [17]. The total number of obtained ECs could be increased by sorting and expansion by another week of culture,

Figure 6 Successful engraftment of generated hPSC-derived EPs to form functional vessels in vivo. (A) Schematic of our finalized approach for generation of hPSC-derived EPs $\left(\mathrm{VEC}^{+} \mathrm{CD} 31^{+} \mathrm{CD} 34^{+} \mathrm{CD} 14^{-}\right)$. This is comprised of phase 1 and 2 in only 6 days, and sequential application of the following molecules: BMP4/GSK-3 $\beta$ I in phase 1 and VEGF-A/DAPT in phase 2. (B) Comparison of cell numbers of hPSCs before differentiation, generated EPs (day 6), and VEC ${ }^{+} \mathrm{CD} 31^{+}$ECs (day 14 ) after phase 3 expansion using our differentiation protocol. (C) Matrigel (MG) plugs with hPSC-derived EPs subcutaneously transplanted into NOD/SCID mice. In vivo live images of capillary vessel formation generated by the hPSC-derived VECEGFP $^{+}$EPs 2 weeks after transplantation. BF, bright field. Scale bars, $100 \mu \mathrm{m}$ (bottom) and $200 \mu \mathrm{m}$ (top). (D) The MG plug with hPSC-derived EPs were harvested 4 weeks after transplantation (top), fixed in $2 \%$ paraformaldehyde (bottom, left), and subjected to fluorescent whole-mount immunostaining. The bottom-right panel shows anastomosis (arrowhead) between the neovessel formed by transplanted EGFP ${ }^{+}$EPs and a host vessel of NOD/SCID mice expressing murine VE-cadherin (red). Scale bars, $500 \mu \mathrm{m}$ (top) and $200 \mu \mathrm{m}$ (bottom, right). (E) Fluorescent immunostaining of cryosections of the MG plug in D shows anastomoses (arrowheads) between the neovessel formed by transplanted EGFP EPs and host capillary vessels of NOD/SCID mice expressing murine VE-cadherin (red). Scale bar, $20 \mu \mathrm{m}$. (F) The neovessels formed by transplanted EGFP $^{+}$EPs in a Matrigel plug with functional anastomosis (arrowhead) to native vessels were labeled with lectin (GS-IB , blue). Scale bar, $20 \mu \mathrm{m}$. (G) In vivo live images of EGFP ${ }^{+}$EP-derived capillary vessels 3 months after transplantation. Black arrowheads indicate host angiogenic sprouting capillary vessels, whereas white arrowheads indicate neovessels formed by transplanted EPs in connection with host sprouting vessels. Scale bars, $100 \mu \mathrm{m}$. (H) The transplanted cell numbers of EPs $\left(V_{E C}^{+} \mathrm{CD} 31^{+} \mathrm{CD} 34^{+} \mathrm{CD} 14^{-}\right)$sorted on day 6 , ECs $\left(\mathrm{VEC}^{+} \mathrm{CD} 31^{+}\right)$sorted on day 14 or VEC-EGFP ${ }^{+}$HUVECs were positively correlated with quantitative total tube area in in vivo Matrigel plugs. EPs have a three- to fivefold efficacy of quantitative vascular tube formation in vivo, compared with ECs or HUVECs. $* P<0.05$ and $* * P<0.01$. 
resulting in a total yield of seven ECs from every hESC in the starting culture, and therefore, a total of 3 weeks of differentiation would be required to generate ECs of a sufficient number for revascularization purposes [2, 5]. Tatsumi et al. [18] have reported that activation of the Wnt signaling pathway by the GSK-3 $\beta$ inhibitor BIO as an initial priming step, combined with VEGF-A administration in a later phase, promoted hESC differentiation into $\mathrm{VEC}^{+}$cells to $20 \%$ of the total number of differentiating cells on day 5 . However, a net yield of ECs $(0.2$ ECs from every hESC) in their approach was still modest, and they did not show in vivo vessel formation by the generated ECs (Supplementary information, Table S2). In contrast, we find that controlled activation or inhibition of four extracellular ligand-receptor systems, the BMP, Wnt, VEGF, and Notch signaling pathways, enabled rapid and more efficient endothelial differentiation. Stepwise modulation of these signaling pathways significantly promoted hPSC differentiation into endothelial lineage cells, specifically $\mathrm{VEC}^{+} \mathrm{CD} 31^{+} \mathrm{CD} 34^{+} \mathrm{CD} 14^{-} \mathrm{EPs}$, through $\mathrm{KDR}^{+}$mesodermal precursors. Our protocol results in $\sim 50 \%$ of the cells being EPs after 6 days of differentiation, corresponding to a total yield of 2.5 EPs for every hPSC in the starting culture. Following further expansion for 8 days after sorting of EPs, corresponding to a total of 14 days of differentiation, the yield was 20 $\mathrm{VEC}^{+} \mathrm{CD} 31^{+}$ECs for every hPSC in the initial culture, and this could be increased further by yet another week in culture to up to 140 ECs for every hPSC in the initial culture, indicating that purified EPs could be expanded without compromising their endothelial identity (Figure 2I and 2J). Thus, our protocol represents a 12- to 20-fold improvement in the yield of ECs compared to previously published results (Supplementary information, Table S2). Importantly, the generated EPs on day 6 could be transplanted for revascularization; they rapidly and successfully formed functional vessels with anastomoses with the host circulation after transplantation in vivo with or without a diseased model (MI) (Figure 6 and Supplementary information, Figure S7), and maintained their endothelial identity and functional properties with beneficial outcomes throughout the time line of our experiments ( $>$ 3 months). Perhaps even more importantly, we provide direct experimental evidence that EPs exhibit markedly improved vessel formation compared to mature ECs obtained at a later phase. Notably, this reduced the required time to obtain sufficient quantities of transplantable cells from hPSCs from 20 [17] to 6 days of differentiation. This is of not only experimental interest but also may be of importance for the field of regenerative medicine, since it has been suggested that shorter differentiation protocols may be beneficial for therapeutic applications
$[1,2,5]$.

An important aspect of our findings is the highefficiency generation of hPSC-derived EPs. Although a stringent definition and the molecular signature of EPs in adult circulation has been still a matter of debate [14, 51], it is generally agreed that they exhibit specific functional features, including capacity to form vessel-like structures that integrate with native vessels of immunocompromised hosts in vivo, and significantly higher proliferative and clonogenic potential compared to mature ECs [12$14,28,32,33,56-59]$. To date, the existence of hPSCderived EPs has been less frequently investigated or debated; however, we hypothesized that there should be a similar EP population in the hPSC-derived endothelial lineage, and demonstrated that $\mathrm{CD} 34^{+} \mathrm{CD} 14^{-}$cells among the hPSC-derived endothelial lineage $\left(\mathrm{VEC}^{+} \mathrm{CD} 31^{+}\right)$on day 6 of differentiation constitutes a discriminative EP population, because the $\mathrm{VEC}^{+} \mathrm{CD} 31^{+} \mathrm{CD} 34^{+} \mathrm{CD} 14^{-}$cells exhibited the highest angiogenic, proliferative, and clonogenic potential (Figures 2, 3 and 6), along with a characteristic angiogenic molecular profile (Figure 2I and 2J), similar to HUCBCs that are considered to likely contain an EP population in adult circulation [5, 12-14, 32-37, 56]. The differentiation potential of the hPSC-derived $\mathrm{VEC}^{+} \mathrm{CD} 31^{+} \mathrm{CD} 34^{+} \mathrm{CD} 14^{-} \mathrm{KDR}^{\text {high }}$ EPs was exclusively restricted to mature, fully differentiated ECs, and formed functional capillaries in vivo. To our knowledge, this is the first report of better identification and purification of hPSC-derived EPs.

Identifying molecules maintaining EPs in a proliferative state provided insights into the molecular circuitry controlling conversion of EPs to mature ECs. VEGF-A is a key angiogenic signal, and VEGF-A/KDR signaling plays a central role in embryonic vasculogenesis and postnatal angiogenesis $[25,60]$. We find that administration of VEGF-A leads to maturation of EPs, with concomitant lower levels of KDR protein. This appears to be mediated by upregulation of Dll4, a ligand for Notch receptors expressed in EPs. Dll4-mediated activation of Notch leads to downregulation of KDR expression, at least in part through direct effects of Notch target genes such as Hes1 and Hey1 on the KDR promoter, as previously shown in the HUVEC study [61]. Inhibition of Notch signaling, either by pharmacological treatment or shRNA-mediated knockdown of key components of the Notch signaling pathway, blocked the attenuation of KDR expression. This in turn maintained the responsiveness of EPs to VEGF-A and kept EPs in a proliferative state.

Notch signaling is an evolutionarily conserved pathway originally identified in Drosophila melanogaster, where Notch receptor activation in a field of neural 
progenitor cells maintains the undifferentiated state in a process known as lateral inhibition [62]. Notch signaling has been dubbed "the gatekeeper of differentiation" in Drosophila neurogenesis, where Notch downstream target genes of the Hairy/Enhancer of Split family inhibits expression of proneural genes. However, a string of publications from mammalian systems have revealed that Notch signaling in some contexts also can promote differentiation, including development of the mammalian neural [63], hematopoietic [64], and vascular systems [65]. In cardiovascular development, Notch signaling has been implicated in numerous processes and differentiation steps, including vascular smooth muscle differentiation, arterial-venous cell fate decisions, proliferation of ECs and angiogenic growth of the blood vessel network [41, 43, 44, 66]. Between ECs, VEGF-A-induced D114/ Notch signaling restricts tip-cell formation and angiogenic sprouting, possibly by repressing the expression of KDR in ECs, and genetic deletion or pharmacological inhibition of Notch signaling enhanced angiogenic sprouting and increased EC proliferation in the retinal vasculature $[41,66]$. We extend these findings by providing experimental evidence that VEGF-induced activation of the Notch signaling pathway attenuates KDR expression in $\mathrm{KDR}^{+}$mesodermal precursors and EPs, permits differentiation of $\mathrm{KDR}^{+}$precursors into non-endothelial lineages (vascular SMCs and cardiomyocytes), and promotes maturation of EPs to mature ECs during hPSC differentiation. Conversely, inhibition of Notch signaling results in maintained high levels of KDR expression in $\mathrm{hPSC}$-derived $\mathrm{KDR}^{+}$precursors and EPs, thereby promoting proliferation at the expense of maturation along the EC lineage. Thus, in EPs, Notch signaling operates as a key mediator of a negative feedback loop that links VEGF activation with maturation to fully differentiated ECs. By uncoupling VEGF stimulation and maturation, inhibition of the Notch pathway permits the stable culture and expansion of EPs, which is achieved mechanistically by alleviating Notch-induced repression of the KDR promoter. Taken together, this Notch-VEGF interaction provides the molecular basis for the efficient generation of EPs in our differentiation protocol (Supplementary information, Figure S8A and S8B).

In summary, this study presents a new efficient approach to generate hPSC-derived EPs. Sequential modulation of four signaling pathways, characterized by BMP4/Wnt-mediated priming of pluripotent cells to early mesoderm, and EP induction and maintenance by VEGF-A administration in combination with inhibition of Notch signaling, promotes hPSC differentiation into EPs with significantly higher efficiency $(\approx 50 \%)$ and in a shorter time (within 6 days) compared to previously published protocols for endothelial differentiation from hESCs [6-9, 17, 18, 53-56]. Moreover, we did not detect any significant differences in the efficiency of endothelial differentiation between different hESC and hiPSC lines. Human PSC-derived EPs generate functional capillary networks when transplanted in vivo, and can be further expanded in vitro. Finally, our study provides data supporting the notion that hPSC-derived EPs are a superior vehicle for therapeutical vascularization compared to more mature populations of ECs in vivo. EPs exhibited markedly improved vessel formation relative to mature ECs upon transplantation, and formed functional anastomoses with the host circulation with better outcomes in a mouse MI model. Tumor formation, a potential concern when transplanting progenitor cell populations with proliferative capacity, was not detected upon grafting of EPs. Conceptually, if patient-derived or allogeneic iPSCs are available, hiPSC-derived EPs generated by our approach might serve as useful therapeutic tools for even acute/subacute ischemic diseases, although further studies are needed. Taken together, our results provide novel insights into the cellular and molecular pathways regulating hPSC differentiation into $\mathrm{EPs} / \mathrm{ECs}$, which may be instrumental in developing novel therapeutic avenues of cellular regeneration for ischemic cardiovascular diseases.

\section{Material and Methods}

\section{Human PSC culture}

The hESC cell lines HUES3 (Harvard University), WA07, and WA09 (both WiCell Research Institute), and hiPSCs [67] were used for this study. The use of these cell lines were approved by the Institutional Review Board (IRB) for human and animal research at Massachusetts General Hospital (MGH), and funding covering the performed experiments was secured from approved non-federal funding resources. Human ESCs/iPSCs were maintained on irradiated mouse embryonic fibroblast (MEF) feeder cells in DMEM/F12 medium (Gibco) supplemented with $20 \%$ KnockOut Serum Replacement (KO-SR; Gibco/LifeTechnologies), $2 \mathrm{mM}$ L-glutamine (Gibco), $0.1 \mathrm{mM}$ non-essential amino acids (Gibco), $0.1 \mathrm{mM} 2$-mercaptoethanol (Gibco), and $10 \mathrm{ng} / \mathrm{ml}$ basic FGF-2 (R\&D). For feeder-free culture of hESCs/hiPSCs prior to endothelial differentiation, cells were passaged onto Matrigel (BD Bioscience) coated plates $(0.3 \mathrm{mg} / \mathrm{mL})$ and cultured in mTeSR-1 medium (StemCell Technologies) according to instructions from the manufacturer. Human fibroblasts, 293FT cells and MEFs were maintained in DMEM containing 10\% fetal bovine serum (FBS), $2 \mathrm{mM} \mathrm{L}$-glutamine, and $1 \mathrm{mM}$ sodium pyruvate.

\section{VEC-GFP hESC reporter line}

A reporter cell line was constructed for monitoring differentiation of pluripotent hESCs to the endothelial lineage. A 2.5-kilobase fragment $(-2486,+24)$ of the human VEC promoter region [15, 16] was isolated and amplified from a bacterial artificial chromo- 
some (BAC) clone by PCR. The fragment was placed upstream of EGFP in a promoterless, $p F U G W$-based lentiviral vector to generate the lentiviral hVEC-EGFP reporter vector. 293FT cells were transfected with hVEC-EGFP along with standard packaging vectors. Viral supernatants were collected in three consecutive days $72 \mathrm{~h}$ after transfection, concentrated by ultracentrifugation, and used to transduce undifferentiated WA09 hESCs. After selection using antibiotic resistance, the transduced hESCs were picked for isolation and expanded to confirm the viral incorporation by PCR. After expansion, these hVEC-EGFP reporter hESCs were differentiated and screened for the presence of cells with co-expression of EGFP and the pan-EC marker PECAM-1 (CD31) or endogenous VEC.

\section{Vascular endothelial differentiation protocols}

Prior to differentiation to the endothelial lineage, hVEC-EGFP hESCs were grown to confluence on Matrigel-coated plate in mTeSR-1 medium as described above. Endothelial differentiation was achieved through modification of two previously described basal protocols [17-21]; one based on the generation of EB formation (method A), and the other using dissociated adherent single cells without EB formation (method B) (Figure 1E and 1F). Using these two protocols, we screened for $>60$ bioactive small molecules (Supplementary information, Table S1) that would promote endothelial differentiation. The molecules were purchased from Peprotech, unless the vendor is specified. In method A, confluent hVEC-EGFP hESCs were incubated in $0.5 \mathrm{mg} / \mathrm{ml}$ dispase (Gibco) at $37{ }^{\circ} \mathrm{C}$ until colonies were completely detached from the substrate. Human ESC-derived EBs were then cultured on ultra-low cluster plates (Costar) in hESC differentiation medium (DMEM/ F12 containing 2\% KO-SR, $2 \mathrm{mM}$ L-glutamine, $0.1 \mathrm{mM}$ nonessential amino acids, and $0.1 \mathrm{mM} 2$-mercaptoethanol), supplemented with $20 \mathrm{ng} / \mathrm{ml} \mathrm{BMP} 4$ (R\&D Systems), at $37{ }^{\circ} \mathrm{C}$ with $5 \%$ $\mathrm{CO}_{2}$ (day 0-3; phase 1). On day 3, EBs are transferred to adherent conditions on Matrigel-coated plates and cultured with $50 \mathrm{ng} /$ ml VEGF (Peprotech) (day 3-7; phase 2). Medium was replaced every other day. In the method B, confluent hVEC-EGFP hESCs were dissociated to single cells using Accutase (Gibco). hESCs were thereafter seeded onto Matrigel-coated plates and cultured in mTeSR-1 medium with $10 \mathrm{ng} / \mathrm{ml}$ of a Rock inhibitor (R\&D) for 1 day. On the next day, medium was changed to DMEM/F12 medium (Gibco) supplemented with N2 and B27 (N2/B27 medium) (Invitrogen) containing $20 \mathrm{ng} / \mathrm{ml} \mathrm{BMP4}$, and the cells were cultured in these conditions for 3 days (day 1-4; phase 1). On day 4, medium was switched to StemPro-34 serum-free medium (Invitrogen) containing $50 \mathrm{ng} / \mathrm{ml} \mathrm{VEGF}$, and the cells were cultured for 2 days (day 4-6; phase 2). On day 7 (method A) or day 6 (method B), adherent differentiated cells were collected and analyzed by FACS. The sorted VEC (EGFP) $)^{+} \mathrm{CD} 31^{+}$endothelial lineage cells were then seeded onto fibronectin (Sigma)-coated plates in endothelial basal medium-2 (EBM-2; Lonza) containing $8 \mathrm{ng} / \mathrm{ml} \mathrm{FGF-}$ 2 and $50 \mathrm{ng} / \mathrm{ml} \mathrm{VEGF}$ and expanded until day 14 (phase 3 ).

\section{FACS analysis and sorting}

Adherent cells were collected from differentiating cultures using $0.25 \%$ trypsin-EDTA and analyzed by FACS (FACSAria; Beckton Dickinson) for detecting the expression of various specific cell surface markers and EGFP expression driven by the lentiviral VEC reporter and for the isolation of endothelial lineage cells.
We used mouse monoclonal fluorochrome-conjugated antibodies against the following human antigens: CD31, VEC, CD14, CD34, KDR, c-kit (BD Bioscience or eBioscience), tumor-related antigen (TRA)-1-60 (Millipore), platelet-derived growth factor receptor $\alpha$ (PDGFR $\alpha$; BioLegend), and cardiac troponin T (cTnT; Abcam). FACS data were analyzed with FlowJo software (Treestart).

\section{Immunocytochemistry}

Cultured adherent cells were fixed with $4 \%$ paraformaldehyde and subjected to immunocytochemistry using standard protocols and the following primary antibodies: anti-CD31 (mouse monoclonal, Cell Signaling Technology), anti-VEC (goat polyclonal, R\&D), anti-vWF (rabbit polyclonal, Abcam), anti-smooth muscle myosin heavy chain (rabbit polyclonal, Biomedical Technologies Inc.), anti- $\alpha$-smooth muscle actin (mouse monoclonal, Sigma), anti-cTnT (rabbit polyclonal antibody, Abcam), anti-vimentin (rat polyclonal, R\&D), and anti-KDR (rabbit polyclonal, Cell Signaling Technology). Alexa-Fluor 488- or 594-conjugated secondary antibodies specific to the appropriate species were used to detect bound primary antibodies (Molecular Probes, 1:500). Nuclei were counterstained with DAPI (Sigma). Images were captured using a DMI6000-AF6000 Leica microscopic imaging system.

\section{In vitro vascular tube formation assay}

$50 \mu 1$ Matrigel per well was placed into wells of a 96-well plate and incubated at $37{ }^{\circ} \mathrm{C}$ for 1 h. $1.5 \times 10^{4}$ of either hPSC-derived endothelial lineage cells sorted by FACS, HUVECs (Cell Applications Inc) or HUCBCs (Harvard University) were suspended in $100 \mu \mathrm{l}$ of EBM-2 supplemented with $5 \%$ FBS and $50 \mathrm{ng} / \mathrm{ml}$ VEGF, and then seeded onto the Matrigel plugs. After $12 \mathrm{~h}$ to 24 $\mathrm{h}$, the endothelial lineage cells formed vascular network on the plugs, which was visualized by phase-contrast microscopy. EGFPnegative cells were stained with fluorescent calcein AM (BD Bioscience). Using a fluorescence microscope (Leica), images in four random fields per well were obtained and processed to measure the vascular length and area quantitatively using imaging analysis software (Image J, NIH). Data was averaged per high-power field. All experiments were performed in quadruplicate.

\section{Cell proliferation assay}

Cell proliferative activity was measured using 3-(4,5-dimethylthiazol-2-yl)-5-(3-carboxymethoxyphenyl)-2H-tetrazolium inner salt (MTS; Promega). The sorted endothelial lineage cells were seeded on 96-well plates in $100 \mu \mathrm{l}$ of EBM-2 containing 2\% FBS with $50 \mathrm{ng} / \mathrm{ml}$ VEGF. At each time point, $20 \mu \mathrm{l}$ of the MTS solution per well was added. After incubation at $37{ }^{\circ} \mathrm{C}$ with $5 \% \mathrm{CO}_{2}$ for $1 \mathrm{~h}$, the absorbance at $490 \mathrm{~nm}$, which is known to correlate with the cell number in the assay [68], was recorded using an ELISA 96-well plate reader. All experiments were performed in quadruplicate.

\section{Quantitative RT-PCR}

Total RNA was isolated from the cells cultured or sorted by FACS using RNeasy mini extraction kit (Qiagen) and reverse-transcribed to cDNA using the iScript cDNA synthesis kit (Bio-Rad). Quantitative real-time RT-PCR was performed on a MasterCycler EP Realplex (Eppendorf) using HotStart-IT SYBR Green Master Mix (Affymetrix) for 40 cycles. Standard deviations of the means in quantitative RT-PCR experiments were obtained from three 
independent experiments. Primer sequences are listed in Supplementary information, Table S3. Threshold cycles of primer probes were normalized to the housekeeping gene GAPDH and translated to relative values.

\section{Gene expression analysis for sorted cell populations}

Human angiogenesis PCR arrays (SABioscieneces) were used to examine the expression profiles of angiogenic genes in the sorted cell populations. Using RNeasy mini extraction kit (Qiagen), total RNA was extracted from hESC-derived EC subpopulations sorted by FACS, including $\mathrm{CD} 34^{+} \mathrm{CD} 14^{-}, \mathrm{CD} 34^{-} \mathrm{CD} 14^{-}$, and $\mathrm{CD}_{1} 4^{+}$cells among $\mathrm{VEC}^{+} \mathrm{CD} 31^{+} \mathrm{ECs}, \mathrm{VEC}^{-} \mathrm{CD} 31^{-}$cells (non-ECs), HUVECs, and HUCBCs, respectively. Clustergram construction and analysis of quantitative PCR data were conducted according to the manufacturer's instructions.

\section{Single-cell clonal assays}

Single cells from subpopulations of hVEC-EGFP hESC-derived endothelial lineages $\left(\mathrm{CD} 34^{+} \mathrm{CD} 14^{-}, \mathrm{CD} 34^{-} \mathrm{CD} 14^{-}\right.$or $\left.\mathrm{CD} 14^{+} \mathrm{ECs}\right)$ were seeded out in a well of a 96-well flat-bottom tissue culture plate precoated with fibronectin using a FACSAria cell sorter (BD Biosciences), and cultured in $200 \mu \mathrm{l}$ EBM-2 media with 2\% FBS and $50 \mathrm{ng} / \mathrm{ml}$ VEGF. To ensure that only one cell had been placed into each well, individual wells were examined under a fluorescence microscope. Cells were then cultured at $37{ }^{\circ} \mathrm{C}$ with $5 \% \mathrm{CO}_{2}$, and media was changed every 3 days. On day 14, each well was examined for the growth of ECs. Wells where two or more ECs were detected using a fluorescence microscope were judged as positive. The number of cells per well was determined by manual inspection using a fluorescence microscope at $100 \times$ magnification. In wells containing more than 100 cells, quantification was performed through trypsinization and manual counting using a hemacytometer.

\section{Western blot}

Total cellular protein was extracted from cultured cells after homogenization in a LP40 lysis buffer containing protease inhibitor and phosphatase inhibitor cocktails (both from Roche). Nuclear protein from cultured cells was obtained through the ProteoExtract Subcellular Proteome Extraction kit (EMD). Protein samples (5 $\mu \mathrm{g}$ ) of nuclear extraction or total lysates were separated by SDSPAGE and transferred onto nitrocellulose membranes (Bio-Rad). Membranes were incubated with primary antibodies to human activated Notch1 (Notch1 intracellular domain) and D114 (Abcam), followed by incubation with horseradish peroxidase-conjugated secondary antibodies (Dako) specific to the appropriate species. Enhanced chemiluminescent (ECL) substrate (SuperSignal West Femto Substrate; Thermo Scientific) was used to detect bound antibody. Bands were visualized onto autoradiography films, scanned and quantified with Image J. The signal intensity was normalized to $\beta$-actin expression.

\section{Knockdown studies}

The shRNA lentiviral constructs targeting Notch1, RBP-JK, Hes1, and Hey1, as well as the control non-targeting shRNA construct, were all obtained from Open Biosystems and subcloned into the pTRIPZ-based lentiviral vector system. Tetracycline-inducible lentiviral particles were assembled by transfecting these shRNA vectors into $293 \mathrm{FT}$ cells along with packaging vectors and used to transduce undifferentiated hVEC-EGFP hESCs. After selection by antibiotic resistance, the transduced hESCs were picked for isolation and expanded. These tetracycline-inducible shRNA hESC lines were then differentiated into endothelial lineages using protocols described above. To obtain the time-dependent knockdown effects for the expression of Notch1, RBP-Jא, Hes1, or Hey1, 1 $\mu \mathrm{g} / \mathrm{ml}$ doxycycline (Sigma) was added to the culture medium at the indicated time point.

\section{Plasmid constructs and dual luciferace assay}

A 1.2-kilobase fragment $(-900,+296)$ of the human KDR promoter region [23] was isolated and amplified from a BAC clone by PCR. The fragment was placed upstream of the firefly luciferase gene in the pGL3 basic plasmid (Promega). The resulting reporter plasmid (pKDR-Luc) was transiently transfected with a control vector (pRL-SV40; Promega) into undifferentiated hESCs using Fugene HD Transfection Reagent (Roche). After 6 h, pKDR-Luctransfected hESCs were stimulated with BMP4 with or without a GSK-3 $\beta$ inhibitor for the next $12 \mathrm{~h}$. After $18 \mathrm{~h}$, luciferase activities were measured using a luminometer (Berthold technologies). Cells were thereafter treated with VEGF-A with or without rhDll4 or DAPT for additional $18 \mathrm{~h}$. Thirty-six hours after the initial transfection, luciferase activities were measured. Firefly luciferase activity was normalized to control Renilla luciferase.

\section{Isolation and culturing of human fetal heart-derived CMCs}

Human fetal hearts at 9 weeks of gestation were obtained from authorized sources. Hearts were cut in small pieces, washed repetitively in solution A (10 mM HEPES, $35 \mathrm{mM} \mathrm{NaCl}, 10 \mathrm{mM}$ glucose, $134 \mathrm{mM}$ sucrose, $16 \mathrm{mM} \mathrm{Na}_{2} \mathrm{HPO}_{4}, 25 \mathrm{mM} \mathrm{NaHCO}_{3}, 7.75$ $\mathrm{mM} \mathrm{KCl}$, and $1.18 \mathrm{mM} \mathrm{KH}_{2} \mathrm{PO}_{4}, \mathrm{pH} 7.4$ ). The first digestion step was performed in solution A supplemented with $0.5 \%$ BSA, 200 $\mathrm{U} / \mathrm{ml}$ collagenase type II (Worthington), and $6 \mathrm{U} / \mathrm{ml}$ protease type XXIV (Sigma) at $37^{\circ} \mathrm{C}$ for $20 \mathrm{~min}$ to remove red blood cells and cell debris. This was followed by four digestion steps performed in solution A supplemented with $400 \mathrm{U} / \mathrm{ml}$ collagenase type II at 37 ${ }^{\circ} \mathrm{C}$ for $20 \mathrm{~min}$ and centrifuged at $30 \times \mathrm{g}$ for $1 \mathrm{~min}$, to separate the $\mathrm{CMC}$ fraction containing $\mathrm{KDR}^{+}$precursors from mature cardiomyocytes [45]. The supernatant containing the CMC fraction was collected, neutralized with FBS, and centrifuged at $1200 \mathrm{rpm}$ for 5 min. The CMC pellet was resuspended in DMEM (high glucose) supplemented with $2 \mathrm{mM}$ L-glutamine, $25 \mathrm{mM}$ HEPES, 10\% newborn calf serum, $5 \% \mathrm{FBS}$, and $1 \%$ pen/strep and cultured on Matrigel-coated 6-well plates with or without DAPT for 4-6 days prior to immunostaining and FACS analyses for the detection of KDR.

\section{In vivo Matrigel plug study}

All experimental procedures and protocols involving animals were approved by the Institutional Animal Care and Use Committee (IACUC) and the IRB for human and animal research at MGH and complied with the Guide for the Care and Use of Laboratory Animals (National Institutes of Health (NIH) publication no. 8623; revised 1985).

To assess the angiogenic potency of the hVEC-EGFP hESCderived endothelial lineage cells to form functionally grafted vasculature in vivo, 1-10 × $10^{6}$ of sorted EPs (day 6) and ECs (day 14) were suspended in a Matrigel solution containing $400 \mu$ l Matrigel and $100 \mu \mathrm{EBM}-2$ supplemented with $10 \% \mathrm{FBS}$ and $100 \mathrm{ng} / \mathrm{ml}$ 
VEGF and injected subcutaneously into the back of immunocompromised mice (NOD/SCID; NCI-Frederick). As a control experiment, the same number of VEC-EGFP ${ }^{+}$HUVECs with Matrigel was injected into the NOD/SCID mice as well. After 2 weeks, the implanted Matrigel plugs were observed in live mice using a fluorescence microscope (Leica), and images in four random fields per plug were obtained and processed to measure the vascular length and area quantitatively using Image $\mathrm{J}$. At the designated time points (2-12 weeks after implantation), the Matrigel plugs are harvested for histological analyses. Separately, we administered intra-vitally isolectin GS-IB 4 from Griffonia simplicifolia (Life Technologies) into a subset of the plug-implanted mice through intravenous injection, followed by the harvest of the plugs for histological analyses as well.

\section{Fluorescent Immunohistochemistry}

The harvested Matrigel plugs were fixed in 4\% paraformaldehyde, processed through a sucrose gradient, and embedded in frozen OCT compound (Tissue Tek). Cryosections ( $8 \mu \mathrm{m}$ thick) were subjected to double immunofluorescence staining using the following primary antibodies: anti-GFP (rabbit polyclonal, Molecular Probes); anti-CD31 (rat monoclonal antibody, BD Bioscience); and anti-mouse VEC (rat monoclonal antibody, R\&D). AlexaFluor 488- and 594-conjugated secondary antibodies specific to the appropriate species were used (Molecular Probes, 1:500). The nuclei were counterstained with DAPI (Sigma).

A subset of the Matrigel plugs were subjected to whole-mount immunostaining according to the instructions previously described [69]. Briefly, the Matrigel plugs were prefixed in 0.1 M PBS with microwave irradiation $(600 \mathrm{~W}, 20 \mathrm{~s})$ and incubated in fixing solution ( $2 \%$ paraformaldehyde) for $1 \mathrm{~h}$ at $4{ }^{\circ} \mathrm{C}$. After washing, immunofluorescent staining of the fixed specimens was performed as described above. All Images were captured using a DMI6000AF6000 Leica microscopic imaging system.

\section{Experimental MI model}

MI was induced in 8-10-week NOD/SCID mice by permanent ligation of the LAD, as previously described [70]. Briefly, mice were anesthetized by intraperitoneal injection of ketamine ( 80 $\mathrm{mg} / \mathrm{kg}$ of body weight) combined with xylazine hydrochloride (16 mg/kg of body weight). After intubation and under mechanical ventilation (MouseVent G500; Kent Scientific Corp.), the heart was exposed through a left thoracotomy, and a suture was placed to ligate the LAD. Immediately after ligation, a total of $1 \times 10^{7}$ hVEC-EGFP hESC-derived EPs in PBS or vehicle (control) was introduced by direct intramuscular injections into the peri-infarcted regions of the myocardium ( $n=5$, respectively). Transthoracic echocardiography (Acuson Sequoia 512 system) was performed with a 14-MHz linear transducer before surgery and 1 and 28 days after MI. Two-dimensional images and M-mode tracings were recorded on the short-axis view at the high papillary muscle level. LVEDD and LVESD were measured for more than 10 beats in a blind fashion. For the assessment of LV function, FS was calculated as follows: (LVEDD - LVESD)/LVEDD × $100(\%)$. Twenty-eight days after MI, the hearts were harvested and fixed in $4 \%$ paraformaldehyde for cryosectioning and immunostaining, as described above.

Separately, survival analysis was performed, and the mice injected with $1 \times 10^{7}$ EPs or vehicle were followed up to 45 days after MI ( $n=14$, respectively).

\section{Statistical analysis}

Data are presented as mean $\pm \mathrm{SD}$. Differences between groups was examined for statistical significance using Student's $t$-test or one-way ANOVA followed by Tukey-Kramer post-hoc test. The survival curves in a MI model were analyzed by the Kaplan-Meier method and compared using the log-rank test. Statistical significance is defined as $P<0.05$.

\section{Acknowledgments}

We would like to thank Kat Folz-Donahue and Laura PrickettRice of the Harvard Stem Cell Institute Flow Cytometry Core Facility for assistance with FACS analysis. We would like to thank Chad Cowan (Harvard University, USA) for iPSCs. We would like to thank all the members of the Chien laboratory for assistance with experiments and helpful discussion. This study was supported by NIH U01H100408 and partially funded by Roche Pharmaceuticals. MS is supported by research fellowships from the Banyu Life Science Foundation International and Uehara Memorial Foundation. EMH is supported by research fellowships from the WennerGren Foundation and Hjärt-Lungfonden.

\section{References}

1 Segers VF, Lee RT. Stem-cell therapy for cardiac disease. Nature 2008; 451:937-942.

2 Bautch VL. Stem cells and the vasculature. Nat Med 2011; 17:1437-1443.

3 Thomson JA, Itskovitz-Eldor J, Shapiro SS, et al. Embryonic stem cell lines derived from human blastocysts. Science 1998; 282:1145-1147.

4 Takahashi K, Tanabe K, Ohnuki M, et al. Induction of pluripotent stem cells from adult human fibroblasts by defined factors. Cell 2007; 131:861-872.

5 Shantsila E, Watson T, Lip GY. Endothelial progenitor cells in cardiovascular disorders. J Am Coll Cardiol 2007; 49:741-752.

6 Levenberg S, Golub JS, Amit M, Itskovitz-Eldor J, Langer R. Endothelial cells derived from human embryonic stem cells. Proc Natl Acad Sci USA 2002; 99:4391-4396.

7 Wang L, Li L, Shojaei F, et al. Endothelial and hematopoietic cell fate of human embryonic stem cells originates from primitive endothelium with hemangioblastic properties. Immunity 2004; 21:31-41.

8 Lu SJ, Feng Q, Caballero S, et al. Generation of functional hemangioblasts from human embryonic stem cells. Nat Methods 2007; 4:501-509.

9 Goldman O, Feraud O, Boyer-Di Ponio J, et al. A boost of BMP4 accelerates the commitment of human embryonic stem cells to the endothelial lineage. Stem Cells 2009; 27:17501759 .

10 Chien KR, Domian IJ, Parker KK. Cardiogenesis and the complex biology of regenerative cardiovascular medicine. Science 2008; 322:1494-1497.

11 Sneddon JB, Borowiak M, Melton DA. Self-renewal of embryonic-stem-cell-derived progenitors by organ-matched mesenchyme. Nature 2012; 491:765-768.

12 Asahara T, Murohara T, Sullivan A, et al. Isolation of putative 
progenitor endothelial cells for angiogenesis. Science 1997; 275:964-967.

13 Urbich C, Dimmeler S. Endothelial progenitor cells: characterization and role in vascular biology. Circ Res 2004; 95:343353.

14 Yoder MC. Is endothelium the origin of endothelial progenitor cells? Arterioscler Thromb Vasc Biol 2010; 30:1094-1103.

15 Gory S, Vernet M, Laurent M, Dejana E, Dalmon J, Huber P. The vascular endothelial-cadherin promoter directs endothelial-specific expression in transgenic mice. Blood 1999; 93:184192.

16 Alva JA, Zovein AC, Monvoisin A, et al. VE-Cadherin-Crerecombinase transgenic mouse: a tool for lineage analysis and gene deletion in endothelial cells. Dev Dyn 2006; 235:759767.

17 James D, Nam HS, Seandel M, et al. Expansion and maintenance of human embryonic stem cell-derived endothelial cells by TGF $\beta$ inhibition is Id1 dependent. Nat Biotechnol 2010; 28:161-166.

18 Tatsumi R, Suzuki Y, Sumi T, Sone M, Suemori H, Nakatsuji N. Simple and highly efficient method for production of endothelial cells from human embryonic stem cells. Cell Transplant 2011; 20:1423-1430.

19 Huber TL, Kouskoff V, Fehling HJ, Palis J, Keller, G. Haemangioblast commitment is initiated in the primitive streak of the mouse embryo. Nature 2004; 432:625-630.

20 Levenberg S, Zoldan J, Basevitch Y, Langer R. Endothelial potential of human embryonic stem cells. Blood 2007; 110:806814.

21 Yang L, Soonpaa MH, Adler ED, et al. Human cardiovascular progenitor cells develop from a KDR+ embryonic-stem-cellderived population. Nature 2008; 453:524-528.

22 Yamaguchi TP, Dumont DJ, Conlon RA, Breitman ML, Rossant J. flk-1, an flt-related receptor tyrosine kinase is an early marker for endothelial cell precursors. Development 1993; 118:489-498.

23 Patterson C, Perrella MA, Hsieh CM, Yoshizumi M, Lee ME, Haber E. Cloning and functional analysis of the promoter for $\mathrm{KDR} / \mathrm{flk}-1$, a receptor for vascular endothelial growth factor. $J$ Biol Chem 1995; 270:23111-23118.

24 Schatteman GC, Dunnwald M, Jiao C. Biology of bone marrow-derived endothelial cell precursors. Am J Physiol Heart Circ Physiol 2007; 292:H1-H18.

25 Yamashita JK, Takano M, Hiraoka-Kanie M, et al. Prospective identification of cardiac progenitors by a novel single cellbased cardiomyocyte induction. FASEB J 2005; 19:1534-1536.

26 Sone M, Itoh H, Yamahara K, et al. Pathway for differentiation of human embryonic stem cells to vascular cell components and their potential for vascular regeneration. Arterioscler Thromb Vasc Biol 2007; 27:2127-2134.

27 Sone M, Itoh H, Yamashita J, et al. Different differentiation kinetics of vascular progenitor cells in primate and mouse embryonic stem cells. Circulation 2003; 107: 2085-2088.

28 Yoon $\mathrm{CH}$, Hur J, Park KW, et al. Synergistic neovascularization by mixed transplantation of early endothelial progenitor cells and late outgrowth endothelial cells: the role of angiogenic cytokines and matrix metalloproteinases. Circulation 2005; 112:1618-1627.

29 Sahara M, Sata M, Morita T, Nakajima T, Hirata Y, Nagai R. A phosphodiesterase-5 inhibitor vardenafil enhances angiogenesis through a protein kinase G-dependent hypoxia-inducible factor-1/vascular endothelial growth factor pathway. Arterioscler Thromb Vasc Biol 2010; 30:1315-1324.

30 Ingram DA, Mead LE, Tanaka $\mathrm{H}$, et al. Identification of a novel hierarchy of endothelial progenitor cells using human peripheral and umbilical cord blood. Blood 2004; 104, 27522760.

31 Yoder MC, Mead LE, Tanaka H, et al. Redefining endothelial progenitor cells via clonal analysis and hematopoietic stem/ progenitor cell principals. Blood 2007; 109:1801-1809.

32 Shantsila E, Watson T, Tse HF, Lip GY. New insights on endothelial progenitor cell subpopulations and their angiogenic properties. J Am Coll Cardiol 2008; 51:669-671.

33 Weiss DJ, Kolls JK, Ortiz LA, Panoskaltsis-Mortari A, Prockop DJ. Stem cells and cell therapies in lung biology and lung diseases. Proc Am Thorac Soc 2008; 5:637-667.

34 Kelly MA, Hirschi KK. Signaling hierarchy regulating human endothelial cell development. Arterioscler Thromb Vasc Biol 2009; 29:718-724.

35 Peichev M, Naiyer AJ, Pereira D, et al. Expression of VEGFR-2 and AC133 by circulating human CD34(+) cells identifies a population of functional endothelial precursors. Blood 2000; 95:952-958.

36 Gao D, Nolan DJ, Mellick AS, Bambino K, McDonnell K, Mittal V. Endothelial progenitor cells control the angiogenic switch in mouse lung metastasis. Science 2008; 319:195-198.

37 Samuel R, Daheron L, Liao S, et al. Generation of functionally competent and durable engineered blood vessels from human induced pluripotent stem cells. Proc Natl Acad Sci USA 2013; 110:12774-12779.

38 Marcelo KL, Goldie LC, Hirschi KK. Regulation of endothelial cell differentiation and specification. Circ Res 2013; 112:1272-1287.

39 Antas VI, Al-Drees MA, Prudence AJ, Sugiyama D, Fraser ST. Hemogenic endothelium: a vessel for blood production. Int $J$ Biochem Cell Biol 2013; 45:692-695.

40 Liu ZJ, Shirakawa T, Li Y, et al. Regulation of Notch1 and Dll4 by vascular endothelial growth factor in arterial endothelial cells: implications for modulating arteriogenesis and angiogenesis. Mol Cell Biol 2003; 23:14-25.

41 Lobov IB, Renard RA, Papadopoulos N, et al. Delta-like ligand 4 (D114) is induced by VEGF as a negative regulator of angiogenic sprouting. Proc Natl Acad Sci USA 2007; 104:3219-3224.

42 Estrach S, Cailleteau L, Franco CA, et al. Laminin-binding integrins induce D114 expression and Notch signaling in endothelial cells. Circ Res 2011; 109:172-182.

43 MacGrogan D, Nus M, de la Pompa JL. Notch signaling in cardiac development and disease. Curr Top Dev Biol 2010; 92:333-365.

44 Grieskamp T, Rudat C, Lüdtke TH, Norden J, Kispert A. Notch signaling regulates smooth muscle differentiation of epicardium-derived cells. Circ Res 2011; 108: 813-823.

45 Qyang Y, Martin-Puig S, Chiravuri M, et al. The renewal and differentiation of Isl1+ cardiovascular progenitors are controlled by a Wnt/beta-catenin pathway. Cell Stem Cell 2007; 1:165-179.

46 Dovey HF, John V, Anderson JP, et al. Functional gamma- 
secretase inhibitors reduce beta-amyloid peptide levels in brain. J Neurochem 2001; 76:173-181.

47 Artavanis-Tsakonas S, Rand MD, Lake RJ. Notch signaling: cell fate control and signal integration in development. Science 1999; 284:770-776.

48 Fischer A, Gessler M. Delta-Notch--and then? Protein interactions and proposed modes of repression by Hes and Hey bHLH factors. Nucleic Acids Res 2007; 35:4583-4596.

49 Lopez AD, Mathers CD, Ezzati M, Jamison DT, Murray CJ. Global and regional burden of disease and risk factors, 2001: systematic analysis of population health data. Lancet 2006; 367:1747-1757.

50 Ben-Shoshan J, George J. Endothelial progenitor cells as therapeutic vectors in cardiovascular disorders: from experimental models to human trials. Pharmacol Ther 2007; 115:25-36.

51 Asahara T, Kawamoto A, Masuda H. Concise review: circulating endothelial progenitor cells for vascular medicine. Stem Cells 2011; 29:1650-1655.

52 Lavoie JR, Stewart DJ. Genetically modified endothelial progenitor cells in the therapy of cardiovascular disease and pulmonary hypertension. Curr Vasc Pharmacol 2012; 10:289299.

53 Nourse MB, Halpin DE, Scatena M, et al. VEGF induces differentiation of functional endothelium from human embryonic stem cells: implications for tissue engineering. Arterioscler Thromb Vasc Biol 2010; 30:80-89.

54 Bai H, Gao Y, Arzigian M, Wojchowski DM, Wu WS, Wang ZZ. BMP4 regulates vascular progenitor development in human embryonic stem cells through a Smad-dependent pathway. J Cell Biochem 2010; 109:363-374.

55 Adams WJ, Zhang Y, Cloutier J, et al. Functional vascular endothelium derived from human induced pluripotent stem cells. Stem Cell Reports 2013; 1:105-113.

56 Losordo DW, Henry TD, Davidson C, et al. Intramyocardial, autologous CD34+ cell therapy for refractory angina. Circ Res 2011; 109:428-436.

57 Ingram DA, Caplice NM, Yoder MC. Unresolved questions, changing definitions, and novel paradigms for defining endothelial progenitor cells. Blood 2005; 106:1525-1531.

58 Schatterman GC, Dunnwald M, Jiao C. Biology of bone marrow-derived endothelial cell precursors. Am J Physiol Heart Circ Physiol 2007; 292:H1-H18.

59 Richardson MR, Yoder MC. Endothelial progenitor cells: quo vadis? J Mol Cell Cardiol 2011; 50:266-272.
60 Lohela M, Bry M, Tammela T, Alitalo K. VEGFs and receptors involved in angiogenesis versus lymphangiogenesis. Curr Opin Cell Biol 2009; 21:154-165.

61 Williams CK, Li JL, Murga M, Harris AL, Tosato G. Up-regulation of the Notch ligand Delta-like 4 inhibits VEGF-induced endothelial cell function. Blood 2006; 107:931-939.

62 Louvi A, Artavanis-Tsakonas S. Notch signalling in vertebrate neural development. Nat Rev Neurosci 2006; 7:93-102.

63 Guruharsha KG, Kankel MW, Artavanis-Tsakonas S. The Notch signalling system: recent insights into the complexity of a conserved pathway. Nat Rev Genet 2012; 13:654-666.

64 Sandy AR, Jones M, Maillard I. Notch signaling and development of the hematopoietic system. Adv Exp Med Biol 2012; 727:71-88.

65 Domigan CK, Iruela-Arispe ML. Recent advances in vascular development. Curr Opin Hematol 2012; 19:176-183.

66 Hellström M, Phng LK, Hofmann JJ, et al. D114 signalling through Notch1 regulates formation of tip cells during angiogenesis. Nature 2007; 445:776-780.

67 Maherali N, Ahfeldt T, Rigamonti A, Utikal J, Cowan C, Hochedlinger K. A high-efficiency system for the generation and study of human induced pluripotent stem cells. Cell Stem Cell 2008; 3:340-345.

68 Cory AH, Owen TC, Barltrop JA, Cory JG. Use of an aqueous soluble tetrazolium/formazan assay for cell growth assays in culture. Cancer Commun 1991; 3:207-212.

69 Takakura N, Yoshida H, Ogura Y, Kataoka H, Nishikawa S, Nishikawa S. PDGFRa expression during mouse embryogenesis: immunolocalization analyzed by whole-mount immunohistostaining using the monoclonal anti-mouse PDGFR a antibody APA5. J Histochem Cytochem 1997; 45: 883-893.

70 Kido M, Du L, Sullivan CC, et al. Hypoxia-inducible factor 1 -alpha reduces infarction and attenuates progression of cardiac dysfunction after myocardial infarction in the mouse. $J$ Am Coll Cardiol 2005; 46:2116-2124.

(Supplementary information is linked to the online version of the paper on the Cell Research website.)

This work is licensed under the Creative Commons Attribution-NonCommercial-No Derivative Works 3.0 Unported License. To view a copy of this license, visit http:// creativecommons.org/licenses/by-nc-nd/3.0 